\title{
Ketohexokinase C blockade ameliorates fructose- induced metabolic dysfunction in fructose-sensitive mice
}

\author{
Miguel A. Lanaspa, ${ }^{1}$ Ana Andres-Hernando, ${ }^{1}$ David J. Orlicky, ${ }^{1}$ Christina Cicerchi, ${ }^{1}$ Cholsoon Jang, ${ }^{2}$ Nanxing Li, ${ }^{1}$ Tamara Milagres, ${ }^{1}$ \\ Masanari Kuwabara, ${ }^{1}$ Michael F. Wempe, ${ }^{3}$ Joshua D. Rabinowitz, ${ }^{2}$ Richard J. Johnson, ${ }^{1}$ and Dean R. Tolan ${ }^{4}$
}

'Division of Renal Diseases and Hypertension, University of Colorado, Aurora, Colorado, USA. ${ }^{2}$ Department of Chemistry and Lewis-Sigler Institute for Integrative Genomics, Princeton University, Princeton, New Jersey, USA. ${ }^{3}$ Department of Pharmacology, University of Colorado, Aurora, Colorado, USA. ${ }^{4}$ Department of Biology, Boston University, Boston, Massachusetts, USA.

\begin{abstract}
Increasing evidence suggests a role for excessive intake of fructose in the Western diet as a contributor to the current epidemics of metabolic syndrome and obesity. Hereditary fructose intolerance (HFI) is a difficult and potentially lethal orphan disease associated with impaired fructose metabolism. In HFI, the deficiency of aldolase B results in the accumulation of intracellular phosphorylated fructose, leading to phosphate sequestration and depletion, increased adenosine triphosphate (ATP) turnover, and a plethora of conditions that lead to clinical manifestations such as fatty liver, hyperuricemia, Fanconi syndrome, and severe hypoglycemia. Unfortunately, there is currently no treatment for HFI, and avoiding sugar and fructose has become challenging in our society. In this report, through use of genetically modified mice and pharmacological inhibitors, we demonstrate that the absence or inhibition of ketohexokinase (Khk), an enzyme upstream of aldolase B, is sufficient to prevent hypoglycemia and liver and intestinal injury associated with HFI. Herein we provide evidence for the first time to our knowledge of a potential therapeutic approach for HFI. Mechanistically, our studies suggest that it is the inhibition of the Khk C isoform, not the $A$ isoform, that protects animals from HFI.
\end{abstract}

\section{Introduction}

Fructose is a major monosaccharide, present in sugars, whose dietary intake has increased over 40 -fold since $1700(1,2)$ especially from the 1970s with the introduction of high fructose corn syrup (HFCS). Added sugars, especially as HFCS, are now found in a wide variety of products, including infant formulas and foods aimed at children (3). Reducing sugar intake is now recommended by multiple agencies including the American Heart Association and the World Health Organization $(4,5)$ because sugar increases the risk for dental caries and metabolic syndrome (4). However, it is very difficult to avoid exposure to HFCS and sucrose in today's culture, since these sugars are commonly added to a great variety of foods.

One patient group that suffers dire consequences from the widespread use of added sugars is individuals with hereditary fructose intolerance (HFI), an autosomal recessive disease with an incidence near 1:30,000, although the frequency may be higher due the difficulty of its diagnosis $(6,7)$. The disease arises from a deficiency in aldolase B activity in the liver, kidney, and small intestine. Subjects with HFI develop severe reactions following fructose ingestion, such as abdominal pain, vomiting, diarrhea, symptomatic hypoglycemia, hyperuricemia, and other pathologies, including death. Newborn infants or babies being weaned from breast milk are at greatest risk, since the autosomal recessive

Authorship note: MAL and AAH contributed equally to this work.

Conflict of interest: MAL, MFW, RJJ, and DRT are members of Colorado Research

Partners LLC, which is developing inhibitors of fructose metabolism for the treatment of metabolic syndrome and kidney disease. RJJ is on the Scientific Board of Amway.

Submitted: April 5, 2017; Accepted: March 8, 2018

Reference information: J Clin Invest. 2018;128(6):2226-2238.

https://doi.org/10.1172/JCI94427. nature of the disease may result in parental ignorance of the disorder (8). In those subjects, acute mortality from fructose exposure may result after hypoglycemia, seizures, lactic acidosis, and coma.

Fructose metabolism in the liver, kidney, and intestine requires the coordinated action of 2 enzymes, ketohexokinase (Khk), which phosphorylates fructose to fructose 1-phosphate (Fru1-P), and aldolase B, which splits Fru1-P into dihydroxyacetone phosphate and glyceraldehyde. In HFI, mutations in the aldolase B gene (aldob) lead to deficiency in aldolase B activity and the accumulation of Fru1-P. This results in marked phosphate and adenosine triphosphate (ATP) depletion and subsequent uric acid generation following fructose ingestion, which are much greater than those observed in healthy individuals (9). The depletion of phosphate sequestered in Fru1-P leads to glycogen accumulation (10) as well as competitive inhibition of glycogen phosphorylase b and phosphoglucomutase by Fru1-P (11). The sum of these alterations is thought to account for the severe acute hypoglycemia and steatohepatitis that develop in individuals with HFI (12-14). Furthermore, these findings have led to the hypothesis that the disease manifestations of HFI might be prevented by blocking the accumulation of Fru1-P by blocking upstream Khk activity (15). In this regard, individuals with essential fructosuria who have a Khk deficiency show no symptoms from ingesting fructose and have a normal life span (16-18).

Recently, an aldolase B knockout (AldoB-KO) mouse was shown to have symptoms similar to HFI (19). In the present report, the hypothesis that blocking Khk activity will prevent HFI in mice was tested using Khk-KO mice. If correct, this hypothesis would open the door for novel therapeutic agents for the disease, for which the only treatment to date (avoidance of fructose) has become almost impossible in our society. 


\section{Results}

Lack of aldolase B is associated with ATP depletion and overactivation of hepatic Khk induced by fructose. The first 2 enzymes in fructose metabolism are Khk and aldolase B. Importantly, unlike other sugar kinases, Khk favors the metabolism of fructose over any other sugar, which is associated with reductions in ATP upon fructose exposure in both human cultured cells and in vivo (20, 21). As a consequence of Fru1-P buildup and reduced ATP levels, inorganic phosphate levels decrease and AMP levels increase, respectively, which activates AMP deaminase 2 (22). In turn, excess AMP enters into the purine degradation pathway, resulting in increased uric acid (Figure 1A). After mice received acute exposure to fructose $(1 \mathrm{~g} / \mathrm{kg}$ for 90 minutes), extracts were made from their dissected livers. Consistent with HFI, AldoB-KO mice showed exacerbated intrahepatic ATP depletion (presumably due to blocked regeneration of ATP from Fru1-P, Figure 1B) and decreased phosphate levels (Figure 1C). Importantly, the overall Khk protein level did not differ markedly among WT, AldoB heterozygous, and AldoB-KO mice (Figure 1D). Hepatic fructosedependent ATP depletion (as a marker for Khk activity), was significantly increased in AldoB-KO mice compared with the other groups (Figure 1E). Moreover, when a similar fructose challenge was given to Khk-KO mice there were no significant changes in hepatic ATP and phosphate levels, suggesting that these changes were part of a mechanism dependent on Khk (Figure 1, B and C).

Ketohexokinase deficiency protects against HFI pathology in AldoB-KO mice. Previously, our group demonstrated that mice lacking aldolase $\mathrm{B}$ are phenotypically identical to individuals with HFI and that after exposure to fructose they are characterized by a high incidence of lethality, inflammation, fatty liver, and growth retardation (19). Since Khk is located upstream to aldolase B in the metabolism of fructose, and Khk is necessary for fructose-dependent ATP depletion and the generation of Fru1-P, the hypothesis that blockade of Khk activity would offer protection from the sequela of HFI was tested. Accordingly, we crossed a Khk-KO mouse with an AldoB-KO mouse to generate an AldoB-KO mouse lacking both isoforms of Khk (A and C) (23, 24). Protein expression in liver extracts from progeny mice confirmed our expectations (Figure 2A). Interestingly, aldolase B heterozygous breeding pairs fed fructose (5\%) demonstrated a significantly reduced percentage of homozygous $A l d o B^{-/-}$pups in the offspring at birth, which was abrogated when the ketohexokinase gene $(k h k)$ was deleted (Figure $2 \mathrm{~B})$.

At weaning, pups bred in the presence of fructose had significantly lower body weights, which indicated a failure to thrive similar to that seen in HFI (Figure 2C) (25). Similarly, after weaning, AldoB-KO mice demonstrated significant weight loss after exposure to various fructose-containing sugars in the drinking water (fructose, sucrose, or HFCS, $5 \% \mathrm{w} / \mathrm{v}$ ), which was not observed in the $\mathrm{Kh}^{-/-} \mathrm{AldoB}^{-/-}$double knockout (Khk/AldoB-DKO) mice (Figure 2D). The drop in body weight loss after exposure of AldoB-KO mice to fructose-containing sugars was paralleled by substantial reduced adipose and muscle weight, suggestive of increased catabolic rates in these tissues. In this regard, epididymal fat as well as the weight of several muscle groups including tibialis anterior, gastrocnemius, and soleus are significantly lower in AldoB-KO than in Khk/ AldoB-DKO mice. Consistently, serum levels of creatinine kinase
(CPK), a marker of muscle breakdown, were significantly elevated in AldoB-KO mice after exposure to fructose (Figure 2, E-G). This observation suggests that Fru1-P accumulation in the liver and other Khk-C-expressing tissues triggers the mobilization of energy stores in distant organs, particularly skeletal muscle. Consistent with published data (22), as a consequence of the absence of Khk activity the Khk-KO mice had a significantly higher excretion of urinary fructose both at baseline and after oral exposure to fructose (Figure $2 \mathrm{H})$. The level of urinary fructose in the Khk/AldoB-DKO mice was 10-fold higher than that in AldoB-KO mice.

Since the majority of dietary sugars, including fructose, are metabolized by the liver and intestines, which coincide with the main sites of expression of Aldob and $\operatorname{Khk}(24,26)$, we analyzed the hepatic and intestinal metabolic effects induced by fructose exposure in AldoB-KO and Khk/AldoB-DKO mice. Histological examination and liver injury scoring analysis (Supplemental Table 1; supplemental material available online with this article; https://doi.org/10.1172/JCI94427DS1) of the livers of AldoB-KO mice demonstrated extensive inflammation after chronic exposure to small amounts of fructose in the chow $(0.3 \%)$ (Figure 3A). The inflammation was characterized by the presence of substantial numbers of both apoptotic and necrotic cells, pigmented macrophages, and overall ductal response with lots of diffuse macrophage infiltration in the parenchyma. Ductal response refers to the reactive processes in disease and injury occurring at the interface of the portal and parenchymal compartments as described by Gouw et al (27) and others (Figure 3A, colored arrows). Consistent with a greater inflammatory phenotype, mRNA levels of both proinflammatory cytokines (Il6 and Tnf $\alpha$ ) and proinflammatory chemokines (Cxcl1) were significantly upregulated in AldoB-KO mice compared with the other groups (Figure 3B). Of interest, minimal or no inflammation was observed in AldoB-KO mice when khk expression was deleted (Figure 3A). Furthermore, consistent with a proinflammatory phenotype, AldoB-KO mice chronically exposed to fructose, but not Khk/AldoB-DKO mice, developed severe hepatic periportal (zone 1) fibrosis as demonstrated with picrosirius red (PSR) staining under polarized light (Figure 3C). The increased fibrosis was paralleled by significantly elevated levels of hepatic hydroxyproline (Figure 3D) and by increased expression levels of fibrotic markers including alpha smooth muscle actin (Asma) and timp1 (Figure 3E). Lastly, AldoB-KO mice treated with fructose demonstrated elevated serum levels of aspartate aminotransferase (AST) and alanine aminotransferase (ALT) (transaminitis), markers of liver inflammation and injury, compared with Khk/ AldoB-DKO animals (Figure 3F). It appears that the observed Khk-dependent leukocyte infiltration in AldoB-KO mice is in response to substantial hepatocyte death. Depletion of intracellular phosphate secondary to its sequestration as Fru1-P and concomitant reduction in ATP through phosphorylation of fructose by Khk results in reduced ATP availability for other cellular processes. These events are further exacerbated in AldoB-KO mice by the absence of proper glycolysis for oxidative phosphorylation and ATP production accompanied by a significant glycogen store disorder. Together, these events substantially predispose AldoB-KO mice hepatocytes to death, thus triggering an inflammatory response characterized by marked leukocyte infiltration. 
A

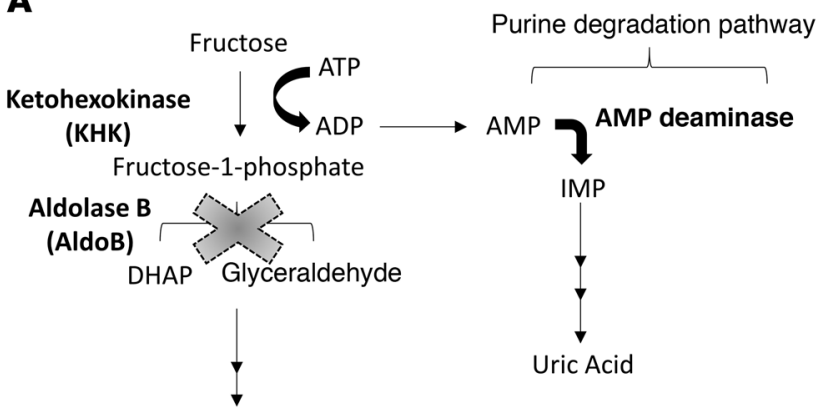

Triglycerides

gluconeogenesis/glycolysis
B

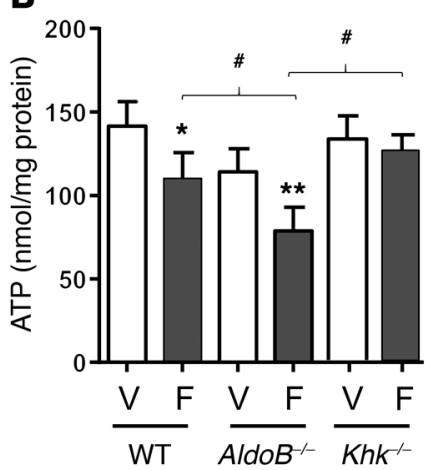

C

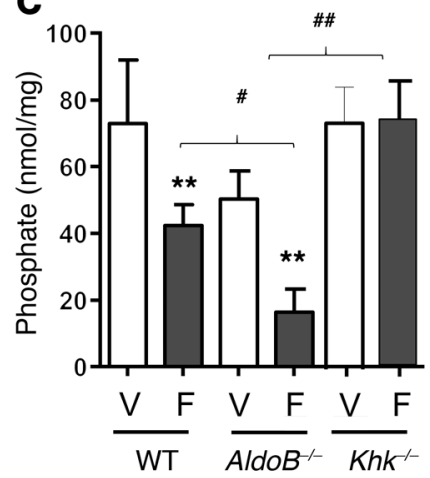

D

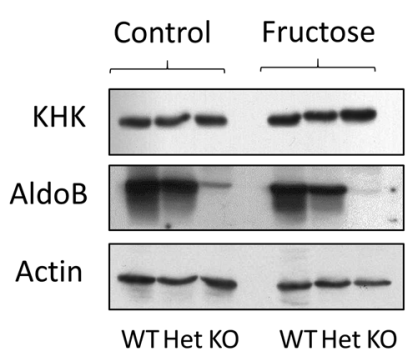

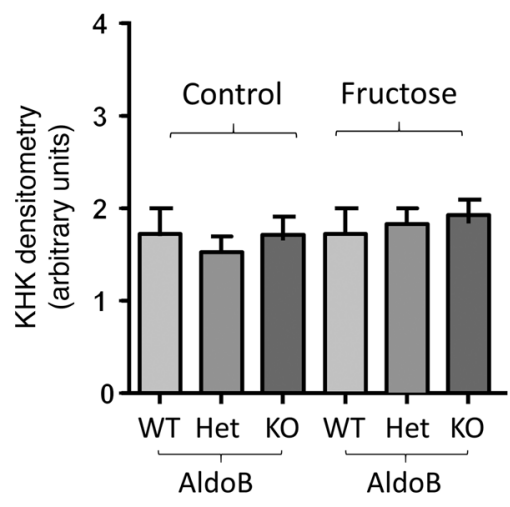

$\mathbf{E}$

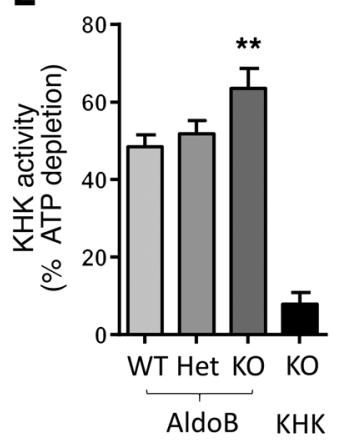

Figure 1. Lack of aldolase B is associated with increased ATP depletion and Khk activation in response to fructose. (A) Schematic describing fructose metabolism and its association with the purine degradation pathway leading to uric acid generation. The loss of the aldolase $B$ gene (indicate by the $X$ ) is associated with Fru1-P accumulation, phosphate and ATP depletion, and increased nucleotide turnover, resulting in uric acid accumulation. (B and $\mathbf{C}$ )

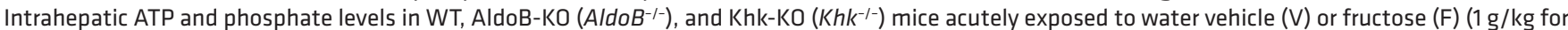
90 minutes). (D) Representative Western blot for aldolase B (AldoB) and ketohexokinase (KHK) in liver extracts from WT, heterozygous (Het), and AldoBKO (KO) mice with or without fructose exposure (1 g/kg for 90 minutes). Densitometry values from $n=15$ animals per group are shown. (E) Ketohexokinase activity in liver extracts from WT (WT AldoB), heterozygous for AldoB-KO (Het AldoB), AldoB-KO (KO AldoB), and Khk-KO (KO Khk) mice. Values were evaluated for statistically significant differences (1-way ANOVA, Tukey post hoc $t$ test analysis, $n=7$ animals per group; ${ }^{*} P<0.05$, ** $P<0.01$ versus respective vehicle controls or ${ }^{\#} P<0.05,{ }^{\#} P<0.01$ versus respective genotypes).

To better understand the mechanism whereby hepatic triglyceride accumulation occurs in aldolase B-deficient mice chronically exposed to fructose, we analyzed the expression of enzymes involved in de novo lipogenesis (fatty acid synthase [FAS], acetylCoA carboxylase [ACC], and ATP-citrate lyase [ACL]) and fatty acid oxidation (carnitine-palmitoyl transferase 1 [CPT1] and enoylCoA hydratase [ECH1]). The baseline expression of CPT1 and ECH1 does not markedly differ among groups fed fructose, whereas AldoB-KO mice have significantly higher levels of FAS, ACC, and ACL, suggestive of an increased ability for de novo lipogenesis (Figure $3 \mathrm{G}$ and Supplemental Figure 1). Interestingly, of these lipogenic enzymes, only the expression of ACL is significantly downregulated when Khk expression is deleted in AldoB-KO mice, suggesting that the marked increased expression of lipogenic enzymes in AldoB-KO mice is independent of fructose metabolism. However, Khk/AldoBDKO mice do not develop fatty liver (see hepatic TG data in Table 1), indicating that despite elevated expression of FAS and ACC in these mice, no increased de novo lipogenesis occurred. This effect could be mediated by increased AMPK-dependent inhibition of ACC in Khk/AldoB-DKO mice as demonstrated by a greater pACC/ACC ratio (Supplemental Figure 1).
One of the major risks associated with fructose consumption in individuals with HFI is the development of hypoglycemic shock (28). Consistent with hypoglycemic shock in individuals with HFI, AldoB-KO mice with acute oral exposure to fructose experienced consistent and severe hypoglycemia in a dose-dependent manner (0.75-2.25 g/kg) (Figure 4A). Importantly, Khk/AldoB-DKO mice that were administered fructose maintained serum glucose, even at the high dose of $1.75 \mathrm{~g} / \mathrm{kg}$ (Figure $4 \mathrm{~B}$ ). This result provided strong evidence that the observed hypoglycemic effect in AldoBKO animals depends directly on active Khk. The mechanism whereby Khk-dependent hypoglycemia occurs in HFI is likely multifaceted and complex. Based on published data, the mechanism would involve a series of molecular events that include impaired glycogenolysis and glycogen accumulation disorders, increased glucokinase-mediated glucose uptake, and reduced de novo glucose production $(10,29,30)$. In this regard, the observation that deletion of Khk prevents the hypoglycemic response to fructose suggests a critical role for Fru1-P in the mechanism.

To examine gluconeogenesis, we evaluated the expression levels of PEPCK and G6Pase, which are known as good indicators of gluconeogenesis (31). However, in opposition to our initial hypoth- 
A

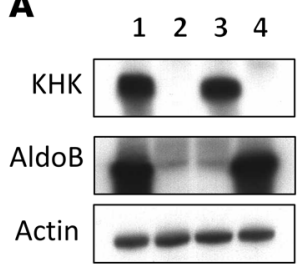

B

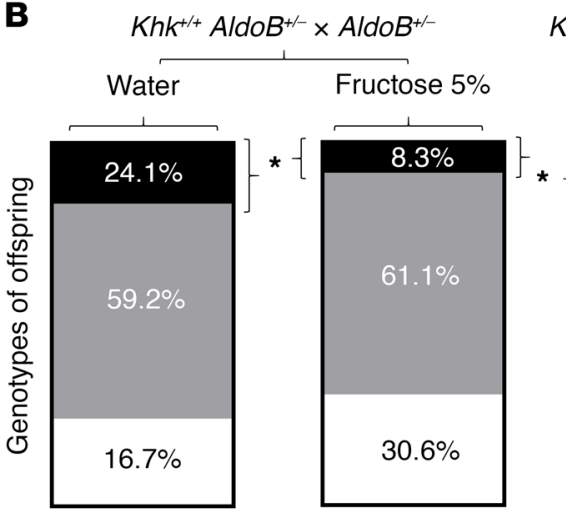

$\mathrm{Khk}^{-1-} \mathrm{AldoB}^{+/-} \times \mathrm{AldoB}^{+-}$

Fructose $5 \%$

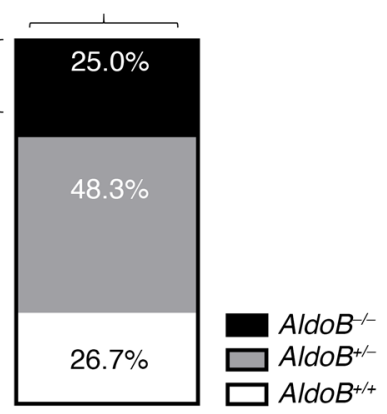

C

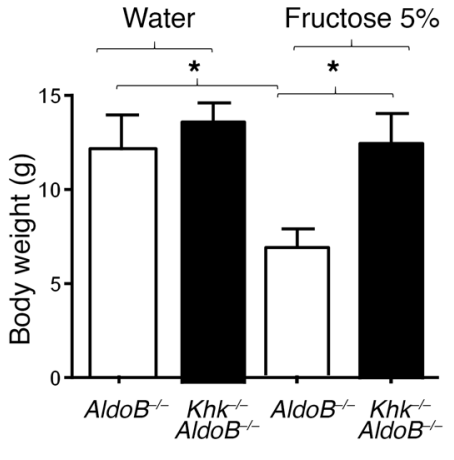

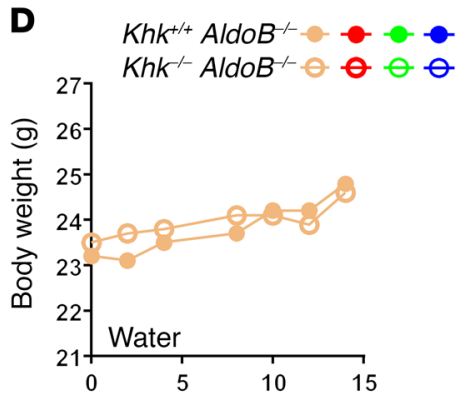

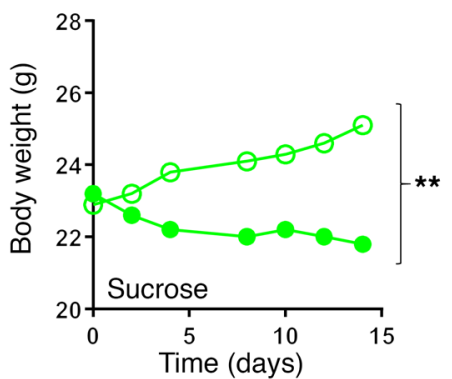

H

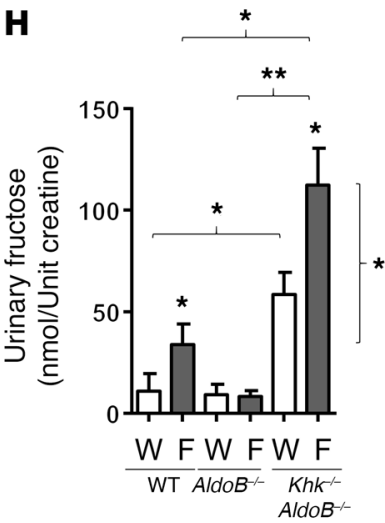

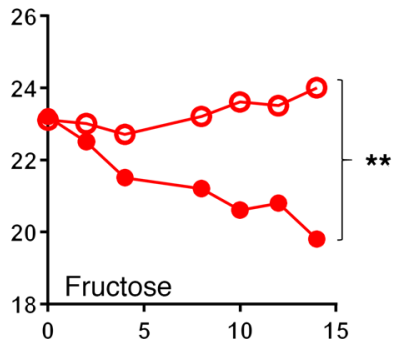
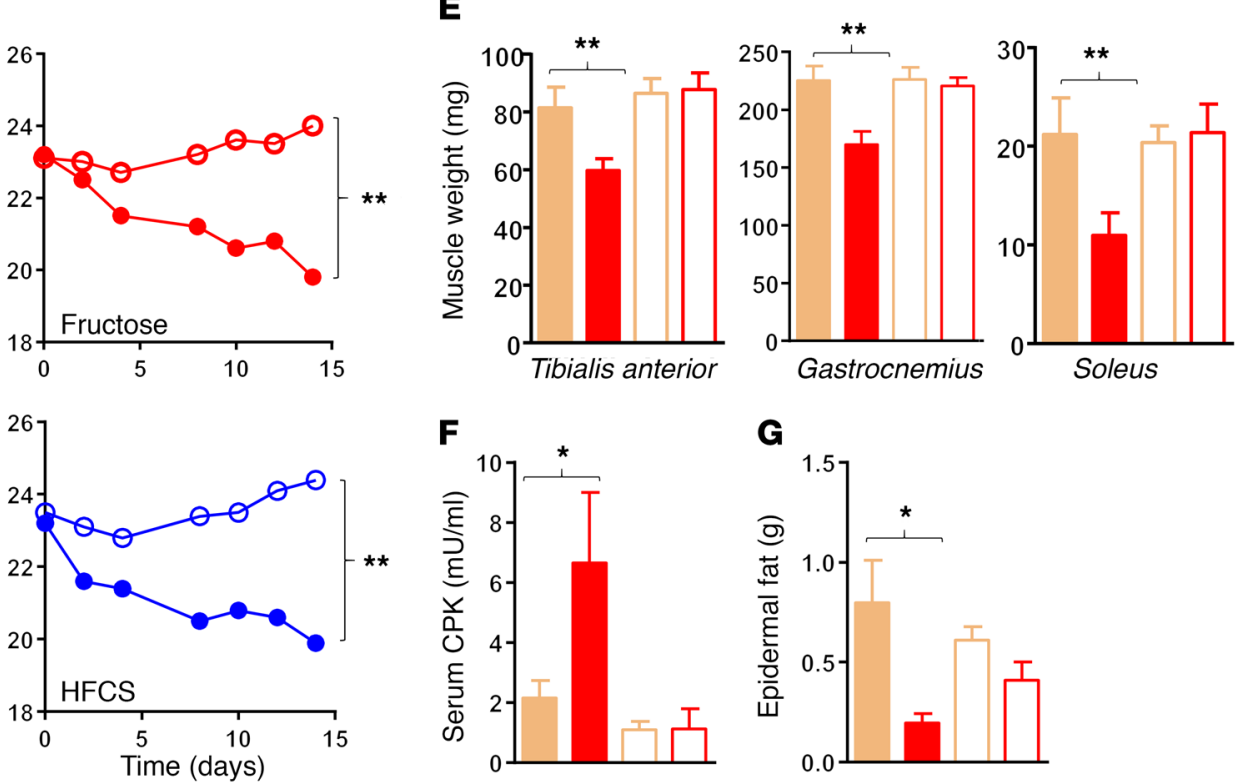

G

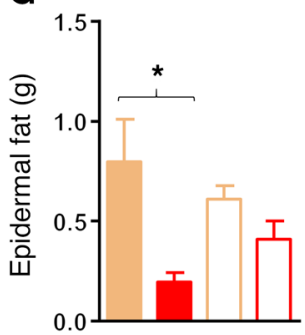

Figure 2. Metabolic responses associated with Khk deletion in AldoB-KO mice. (A) Representative Western blot for aldolase B (AldoB) and ketohexokinase (Khk) in WT (lane 1), Khk/AldoB-DKO (lane 2), AldoB-KO (Iane 3), and Khk-KO (lane 4) mice. (B) Distribution of different genotypes in offspring from breeding aldob heterozygous $\left(A l d o B^{+/-}\right)$pairs exposed to water control or $5 \%$ fructose in the drinking water with either WT $\left(\mathrm{Kh}^{+\mathrm{I}^{+}}\right.$) (left and center) or Khk-KO background ( $\mathrm{Kh}^{-{ }^{-}}$) (right) ( $n \geq 15$ pups analyzed from at least 3 offspring per breeding pair). Total pups born in each group: left graph, WT (13/54), heterozygous (32/54), and knockouts (9/54); center graph, WT (11/36), heterozygous (22/36), and knockouts (3/36); right graph, WT (15/60), heterozygous (29/60), and knockouts (16/60). Statistical significance determined by 2-tailed $\chi^{2}$ test. (C) Average body weight at weaning of AldoB-KO mice (white) or Khk/AldoB-DKO mice $\left(\mathrm{Khk}^{-1-} \mathrm{AldoB}^{-1-}\right)$ (black) from heterozygous breeding pairs. Statistical significance determined by 1-way ANOVA, Tukey's post hoc $t$ test. (D) Changes in body weight of 8-week-old AldoB-KO $\left(\mathrm{Khk}^{+/+} \mathrm{AldoB}^{-I^{-}}\right)$(solid circles) or Khk/AldoB-DKO ( $\mathrm{Kh}^{-{ }^{--}}$Aldo ${ }^{-I^{-}}$) (open circles) mice exposed to the indicated fructose-containing sugars $(5 \% \mathrm{~W} / \mathrm{v})$ for 14 days. Statistical significance determined by 2-tailed $t$ test $\left.{ }^{* *} P<0.01\right)$.(E-C) Muscle weight, serum CPK, and epididymal fat weight at day 15 after water (brown bars) or fructose (red bars) exposure in AldoB-KO (solid bars) and Khk/AldoB-DKO mice (open bars). For E, statistical significance was determined by 1-way ANOVA, Tukey's post hoc $t$ test. $(\mathbf{H})$ Urinary fructose excretion, normalized to units

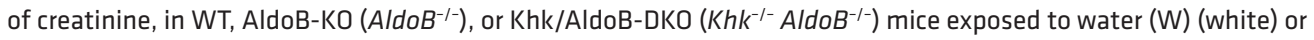
$5 \%$ fructose $W / v(F)$ (gray) for 24 hours. Statistical significance was determined by 1-way ANOVA, Tukey's post hoc $t$ test. ${ }^{*} P<0.05,{ }^{* *} P<0.01(n=7$ animals per group $[\mathbf{C}-\mathbf{H}])$.

esis, hepatic levels of PEPCK and G6Pase are not downregulated in AldoB-KO mice, but rather are higher than those observed in WT mice (Figure 4C and Supplemental Figure 1). Interestingly, the upregulation of these key gluconeogenic enzymes is not prevented, although it is substantially ameliorated in Khk/AldoBDKO mice. It is possible that the elevation of PEPCK and G6Pase in AldoB-KO mice does not necessarily mean increased gluconeogenesis in these animals and could be the consequence of a compensatory mechanism secondary to reduced flux. Therefore, to further evaluate whether increased PEPCK and G6Pase expression in AldoB-KO mice was really associated with an enhanced ability for gluconeogenesis, we performed a pyruvate tolerance 
A

$\mathrm{Khk}^{+/+} \mathrm{AldoB} /$

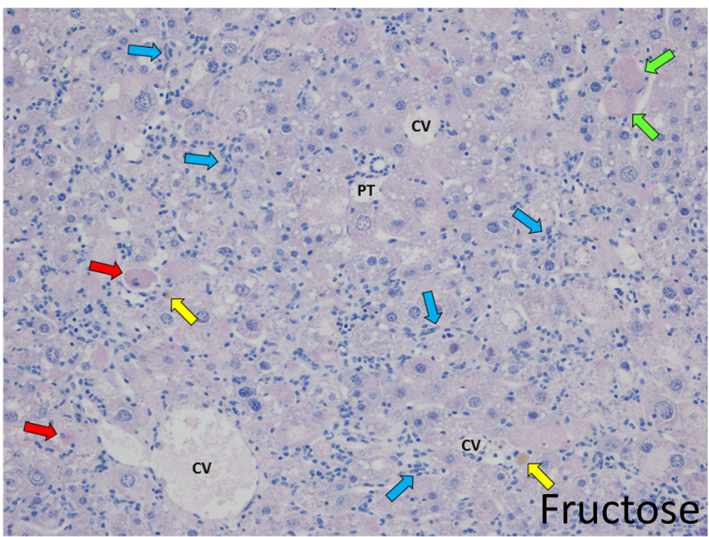

$\mathrm{Khk}^{\prime-} \mathrm{AldoB}$

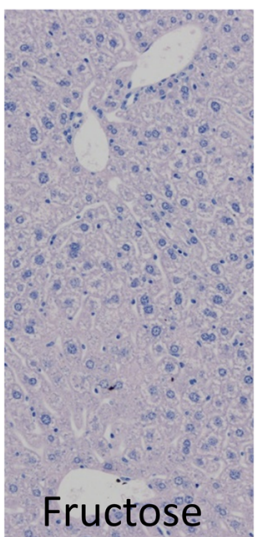

B

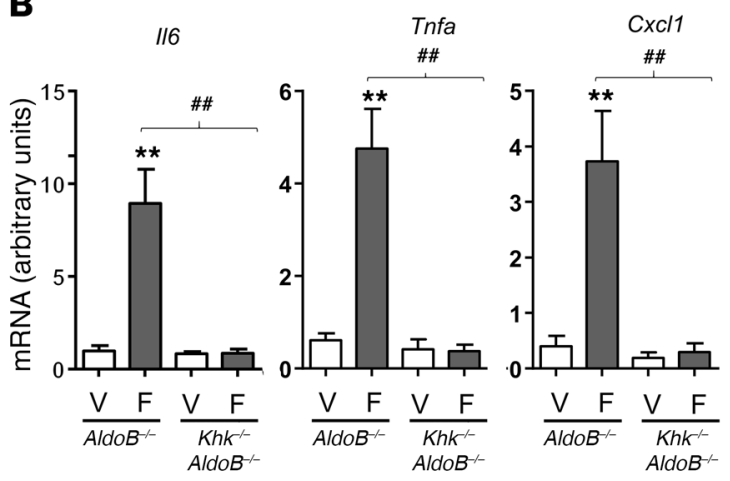

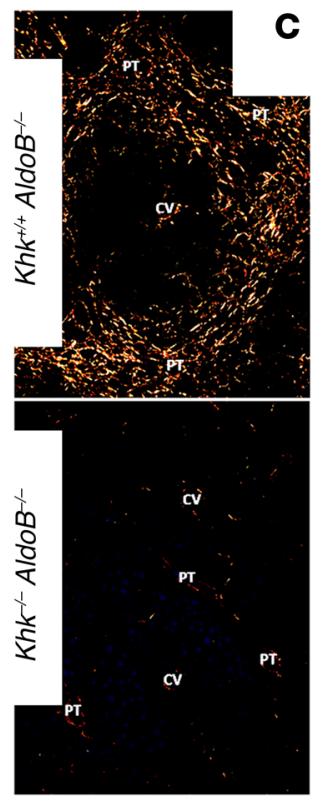

PSR

ave/image

$\times 1000$

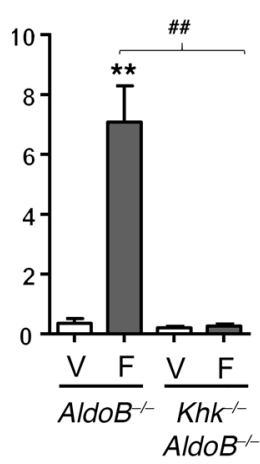

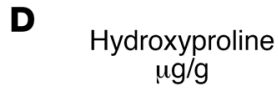

$\mathbf{E}$
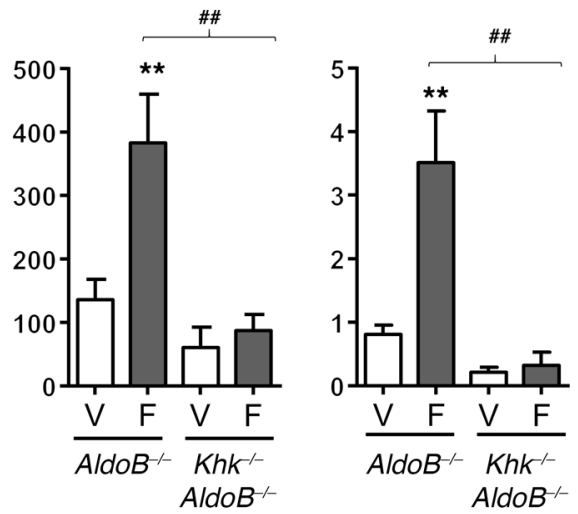

\section{G}

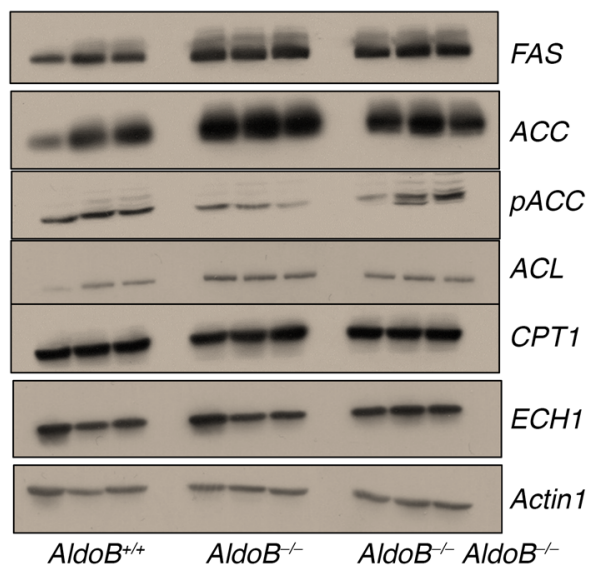

$\mathbf{F}$

AST (U/L)

$\operatorname{ALT}(\mathrm{U} / \mathrm{L})$
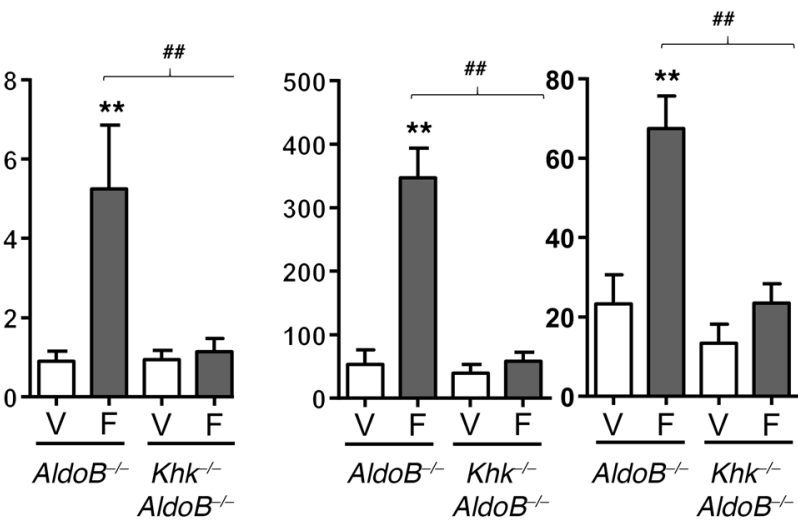

Figure 3. Reduced hepatic inflammation and fibrosis in Khk/ AldoB-DKO mice compared with AldoB-KO mice exposed to

fructose. (A) Representative H\&E images from livers of AldoB-KO $\left(\mathrm{Khk}^{+/+} \mathrm{AldoB^{-/- }}\right.$ ) exposed to $0.3 \%$ fructose in the chow (left), or Khk/ AldoB-DKO ( $\left.\mathrm{Kh}^{-1-} \mathrm{AldoB}^{-1-}\right)$ mice identically exposed to the same chow. Red arrows indicate apoptotic cells, green arrows indicate necrotic cells, yellow arrows indicate pigmented macrophages, blue arrows indicate ductal response and lots of diffuse macrophage inflammation. CV, central vein; PT, portal triad. (B) mRNA levels of proinflammatory cytokines (I/6 and Tnfa) and chemokines (Cxc/7) in mice exposed to sucrose-free (V) or fructose-containing (F) chow. (C) Liver picrosirius red (PSR) staining pictured under polarized light and positive pixel signal denoting increased fibrosis in AldoB-KO mice compared with Khk/AldoB-DKO mice exposed to fructose. CV and PT are as indicated in A. (D-F) Liver hydroxyproline, mRNA levels of fibrotic markers asma and timp1, serum AST, and serum ALT levels in AldoB-KO mice or Khk/AldoB-DKO mice as denoted in A, exposed to sucrose-free chow (V) or fructose (F). (C) Representative Western blot for lipogenic (Fas, Acc and Ser69 phosphorylated ACC, and Acl) and fat oxidation-related enzymes (Cpt1 and Ech1) in low $(0.3 \%)$ fructose-fed mice. Values were evaluated for statistically significant differences ( $n=7$ animals per group, 1-way ANOVA, Tukey's post hoc $t$ test; ${ }^{*} P<0.05$, ${ }^{* *} P<0.01$ versus respective vehicle controls or $\# P<0.05, \# \#<0.01$ versus respective genotypes). 48. Original magnification: $\times 100(A) ; \times 40$ (C).

test. As shown in Figure 4D, and consistent with previous reports in individuals with $\mathrm{HFI}(28,32)$, AldoB-KO mice are able to produce glucose from pyruvate. However, their ability to produce glucose endogenously is substantially blunted compared with WT mice. Furthermore, an intermediate effect in glucose production was observed in Khk/AldoB-DKO mice. The benefit observed in Khk/AldoB-DKO mice could be due to not only an improved gluconeogenic rate compared with AldoB-KO mice, but also to 
Table 1. Biochemical blood and liver parameters in WT, AldoB-KO, Khk/AldoB-DKO, and Khk-A/AldoB-DKO mice exposed to water or fructose

\begin{tabular}{|c|c|c|c|c|c|c|c|c|c|}
\hline \multirow[b]{2}{*}{$\begin{array}{l}\text { Biochemical } \\
\text { blood analysis }\end{array}$} & \multicolumn{2}{|c|}{ WT } & \multicolumn{2}{|c|}{ AldoB-KO } & \multicolumn{2}{|c|}{ Khk/AldoB-DKO } & \multicolumn{2}{|c|}{ Khk-A/AldoB-DKO } & \multirow[b]{2}{*}{$\begin{array}{l}\text { AldoB-KO vs Khk/ } \\
\text { AldoB-DKO fructose } \\
\text { (ANOVA) }\end{array}$} \\
\hline & $\begin{array}{l}\text { Water } \\
(n=5)\end{array}$ & $\begin{array}{c}\text { Fructose } \\
(n=5)\end{array}$ & $\begin{array}{l}\text { Water } \\
(n=5)\end{array}$ & $\begin{array}{c}\text { Fructose } \\
(n=5)\end{array}$ & $\begin{array}{l}\text { Water } \\
(n=5)\end{array}$ & $\begin{array}{c}\text { Fructose } \\
(n=5)\end{array}$ & $\begin{array}{l}\text { Water } \\
(n=5)\end{array}$ & $\begin{array}{c}\text { Fructose } \\
(n=5)\end{array}$ & \\
\hline Clucose (AUC over $90 \mathrm{~min}$ ) & $4.8 \pm 0.3$ & $4.6 \pm 0.3$ & $4.4 \pm 0.3$ & $3.0 \pm 0.2^{\mathrm{A}}$ & $4.8 \pm 0.3$ & $4.9 \pm 0.3$ & $4.5 \pm 0.2$ & $2.0 \pm 0.7^{A}$ & $P<0.01$ \\
\hline Insulin (ng/ml) & $0.62 \pm 0.1$ & $0.46 \pm 0.2$ & $0.46 \pm 0.2^{x}$ & $0.55 \pm 0.1$ & $0.68 \pm 0.1$ & $0.68 \pm 0.1$ & $0.57 \pm 0.1$ & $0.66 \pm 0.3$ & NS \\
\hline Magnesium (mM) & $2.0 \pm 0.4$ & $2.2 \pm 0.3$ & $2.2 \pm 0.3$ & $2.8 \pm 0.2^{\mathrm{A}}$ & $2.0 \pm 0.3$ & $2.0 \pm 0.2$ & $2.2 \pm 0.1$ & $2.6 \pm 0.1^{A}$ & $P<0.01$ \\
\hline Fructose (mM) & $0.1 \pm 0.1$ & $0.7 \pm 0.3^{\mathrm{B}}$ & $0.3 \pm 0.1$ & $1.4 \pm 0.2^{\mathrm{A}}$ & $0.5 \pm 0.2$ & $1.7 \pm 0.2^{\mathrm{A}}$ & $0.2 \pm 0.1$ & $0.8 \pm 0.3^{\mathrm{A}}$ & NS \\
\hline AST (u/l) & $36 \pm 10$ & $35 \pm 9$ & $48 \pm 11^{c}$ & $122 \pm 16^{A}$ & $32 \pm 6$ & $30 \pm 6$ & $49 \pm 9$ & $140 \pm 27^{A}$ & $P<0.01$ \\
\hline ALT (u/l) & $22 \pm 8$ & $25 \pm 10$ & $32 \pm 6^{c}$ & $68 \pm 10^{A}$ & $21 \pm 4$ & $19 \pm 4$ & $30 \pm 8$ & $89 \pm 18^{A}$ & $P<0.01$ \\
\hline \multicolumn{10}{|l|}{ Biochemical liver analysis } \\
\hline Phosphate (nmol/mg) & $71.3 \pm 19.6$ & $40.8 \pm 9.4^{A}$ & $53.8 \pm 6.9^{c}$ & $22.2 \pm 2.8^{A}$ & $88.6 \pm 7.3$ & $85.2 \pm 14.3$ & $70.6 \pm 2.3$ & $38.1 \pm 3.3^{A}$ & $P<0.01$ \\
\hline Uric acid ( $\mu \mathrm{g} / \mathrm{mg})$ & $11.8 \pm 2.9$ & $18.6 \pm 5.2$ & $16.6 \pm 2.2$ & $45.3 \pm 14.3^{A}$ & $7.8 \pm 1.1$ & $6.2 \pm 3.3$ & $14.4 \pm 4.4$ & $60.8 \pm 8.1^{A}$ & $P<0.01$ \\
\hline 6-PG (total ion count $\left.{ }^{*} 1,000\right)$ & $11.5 \pm 1.9$ & $10.9 \pm 3.3$ & $3.7 \pm 0.9^{c}$ & $1.5 \pm 0.8^{A}$ & $3.0 \pm 0.2$ & $5.1 \pm 0.1^{A}$ & & & $P<0.01$ \\
\hline S7P (total ion count ${ }^{*} 1,000$ ) & $64.4 \pm 22.3$ & $80.0 \pm 8.3$ & $42.6 \pm 4.5$ & $8.8 \pm 6.1^{A}$ & $39.8 \pm 4.7$ & $69.9 \pm 11.9$ & & & $P<0.01$ \\
\hline
\end{tabular}

Mice were given a dose of $1.75 \mathrm{~g} / \mathrm{kg}$ fructose, and liver and serum were collected after 90 minutes. Liver parameters were normalized to the milligram of liver protein. Statistical analysis was performed on all the genotypes dosed with fructose using ANOVA coupled with Tukey's post hoc $t$ test. ${ }^{A} P<0.01$, ${ }^{B} P$ $<0.05$. Indicates analysis of significance between AldoB-KO and Khk/AldoB-DKO mice on fructose. Bold indicates statistically significant values versus water in each group. AST, aspartate transaminase; ALT, alanine transaminase; 6-PG, 6-phosphogluconate; S7P, sedoheptulose 7-phosphate.

improved glycogenolysis. Despite some gluconeogenic ability, 18-hour fasting serum glucose levels were substantially lower in AldoB-KO mice compared with WT and Khk/AldoB-DKO mice, although the levels remained relatively normal, which suggests some ability for de novo glucose production in these animals (119 $\mathrm{mg} / \mathrm{dl}$ WT vs $96 \mathrm{mg} / \mathrm{dl}$ KO vs $112.3 \mathrm{mg} / \mathrm{dl} \mathrm{DKO})$.

In individuals with HFI, hepatomegaly is believed to be a consequence of increased glycogen accumulation, and it results in markedly impaired glucose availability and homeostasis. Production and degradation of glycogen are tightly regulated by controlling the activity of glycogen synthase (GS) and glycogen phosphorylase (GYPL), respectively. Activity of these enzymes is maintained by phosphorylation. AMPK phosphorylates and inhibits GS whereas PKA phosphorylates and activates GYPL. Glycogen levels and GS activity are significantly elevated in AldoB-KO mice compared with WT animals (Figure 4E, Supplemental Figure 1 and Table 1). It is important to note that both glycogen accumulation and the continuous activation of GS are prevented in Khk/AldoBDKO mice, suggesting that, unlike AldoB-KO mice, WT and DKO animals could potentially maintain improved glucose homeostasis by mobilizing the glycogen reservoir in the liver.

Glucose uptake is stimulated upon energy demand by cells. In a fed state, insulin promotes glucose uptake by insulin-sensitive tissues. In the liver, besides insulin action, the release of glucokinase from the nucleus to the cytosol is an important step to stimulate glucose uptake for glycolysis. Thus, one possibility is that HFI is associated with an exacerbated glucokinase-mediated response, leading to severe hypoglycemia after a fructose challenge. In this regard, our data show that fructose exposure is not associated with greater insulin release over time (Table 1), which is consistent with previous observations regarding the lack of an active fructose transporter (GLUT5) in pancreatic islets and the failure of fructose to stimulate the release of insulin by pancreatic $\beta$ cells (33). In contrast to the minimal effect of fructose on the release of insulin, hepatic glucokinase expression and location markedly differ between AldoB-KO mice and the other groups. Our data (Figure $4 \mathrm{~F}$ ) demonstrate that AldoB-KO mice have markedly greater baseline expression of glucokinase and fructose-dependent cytosolic release from the nucleus than WT or Khk/AldoB-DKO mice. At 90 minutes after fructose load, cytosolic glucokinase levels are 2.6- and 1.7-fold greater in AldoB-KO mice than in WT or DKO animals, respectively. Fru1-P has been shown to disrupt the bond between glucokinase and its regulatory protein in the nucleus, thus allowing for its cytosolic translocation $(34,35)$. Our metabolomics data demonstrate that AldoB-KO mice have significantly greater accumulation of Fru1-P than WT and DKO mice (Figure $4 \mathrm{G})$. It appears that glucose taken up by AldoB-KO mice is directed toward glycogen accumulation. As described above, glycogen synthase activity is not inhibited in AldoB-KO mice, and glycogen levels are consistently substantially higher in these mice after a fructose challenge when compared with the other groups (Table 1). Analysis of the pentose phosphate pathway (an alternative pathway to glycolysis) indicates that there is a markedly reduced level of metabolites from this pathway in AldoB-KO mice, as identified by substantially lower levels of 6-phosphogluconate and sedoheptulose 7-phosphate. This is consistent with preferential 
A

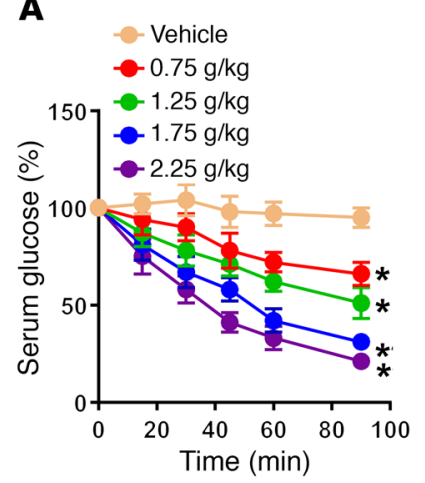

D

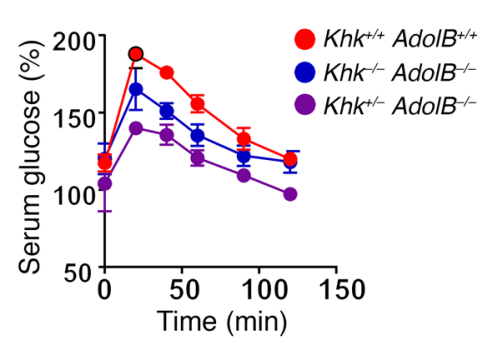

G

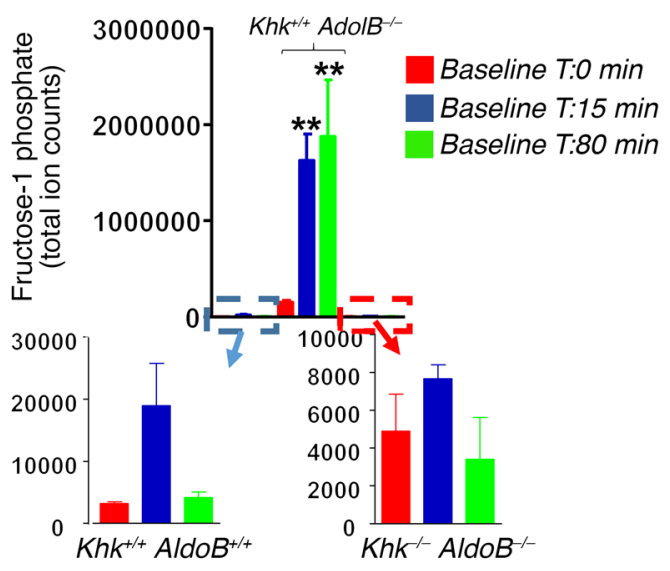

C

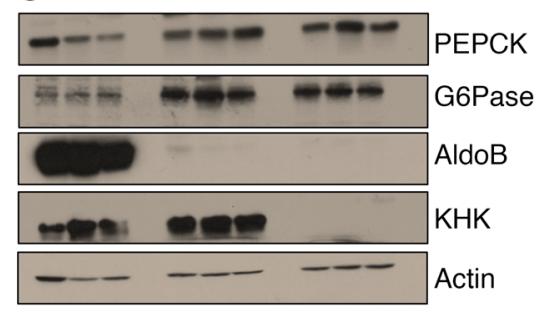

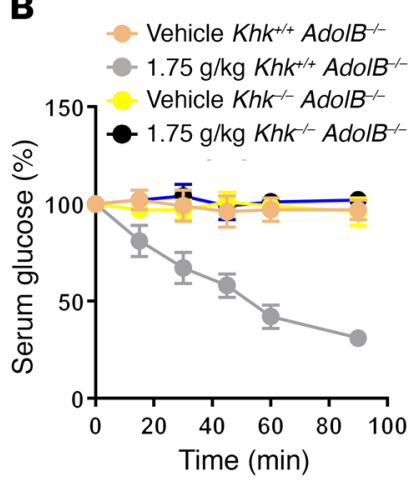

$\mathbf{E}$

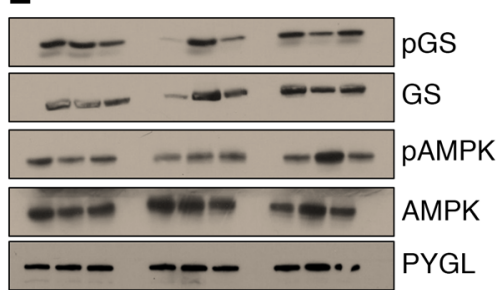

$\mathbf{F}$

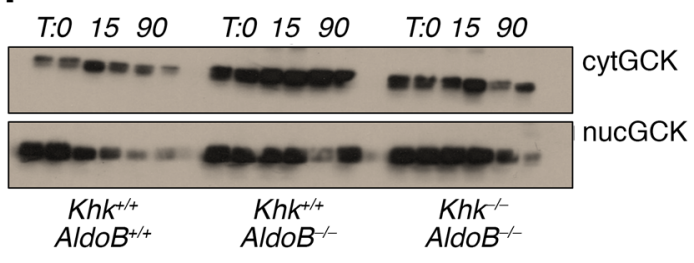

Figure 4. Fructokinase deficiency protects against fructose-induced severe hypoglycemia and metabolic imbalances in AldoB-KO mice. (A) Time course of serum glucose levels in AldoB-KO mice acutely exposed to increasing levels of oral fructose. (B) Time course

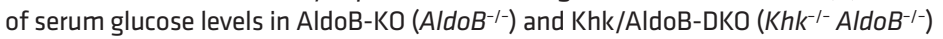
mice acutely exposed to fructose $(1.75 \mathrm{~g} / \mathrm{kg}$ ) or water (vehicle) control. (C) Representative Western blot for gluconeogenic enzymes PEPCK and G6Pase in low (0.3\%) fructose-fed WT (left), AldoB-KO (center), and Khk/AldoB-DKO (right) mice. (D) Serum glucose levels after pyruvate tolerance test in the same groups as in C. (E) Representative Western blot for glycogen synthase (GS) total and inhibited (pGS) as well as total and active AMPK and total glycogen phosphorylase (PYCL) in the same groups as in C. (F) Representative Western blot for cytosolic and nuclear glucokinase expression at baseline and 15 and 90 minutes after fructose challenge in the same groups as in C. (C) Intrahepatic Fru1-P levels at baseline and 15 and 90 minutes after fructose challenge in the same groups as in $\mathbf{C}$. $(\mathbf{H})$

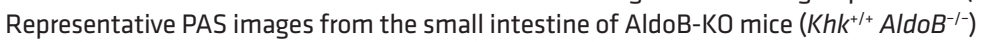
(left and center) or Khk/AldoB-DKO mice $\left(\mathrm{Khk}^{-1-} \mathrm{AldoB^{-1 }}\right.$ ) (right) collected 90 minutes after exposure to oral fructose $(1.75 \mathrm{~g} / \mathrm{kg}$ ). The destruction in the tip of the papilla (green arrows) and luminal apoptotic cells (blue arrows) in the jejunum area are indicated. Values were evaluated for statistically significant differences ( $n=7$ animals per group, 1-way ANOVA, Tukey's post hoc $t$ test; ${ }^{*} P<0.05$, ${ }^{* *} P<0.01$ versus respective vehicle controls).

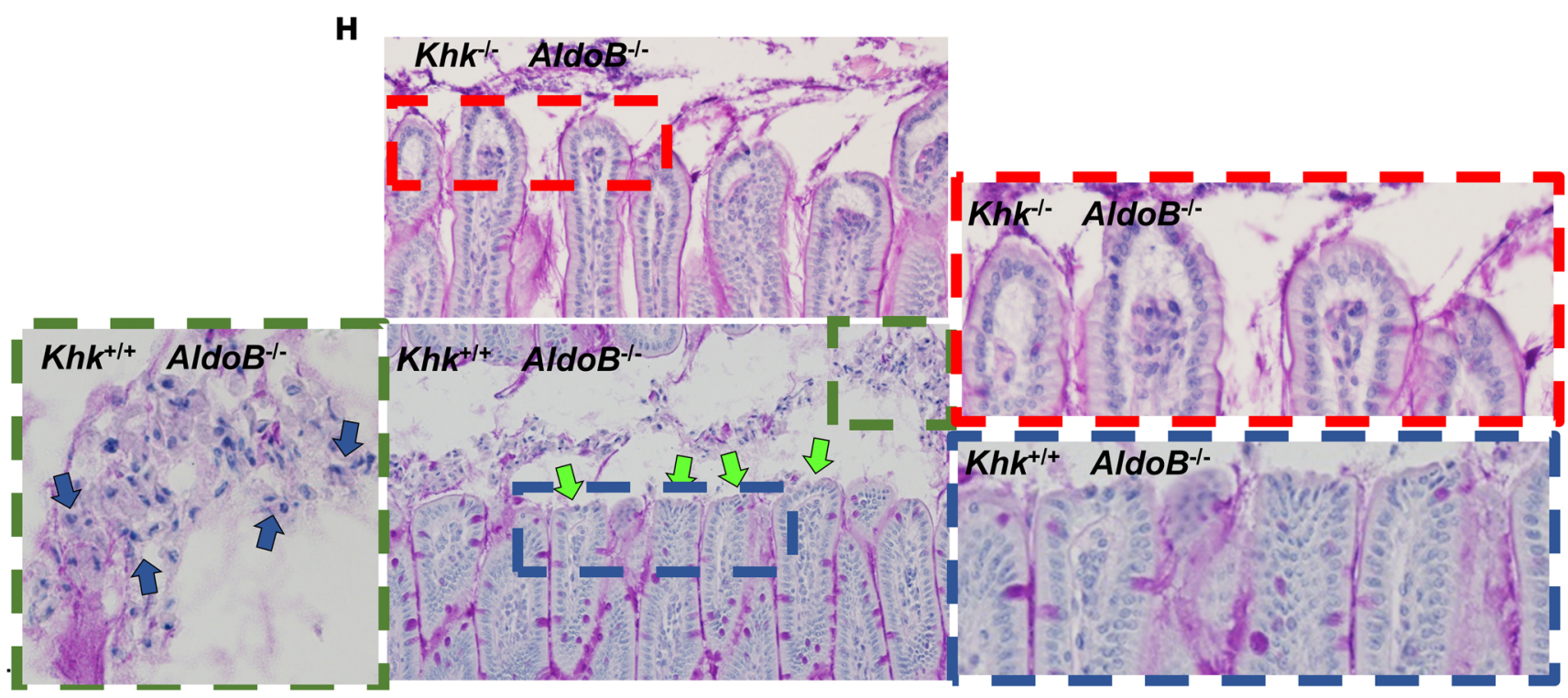


A

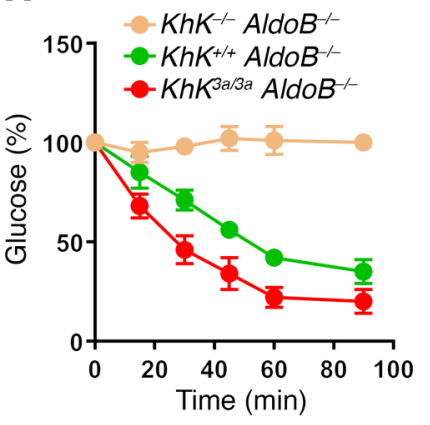

B

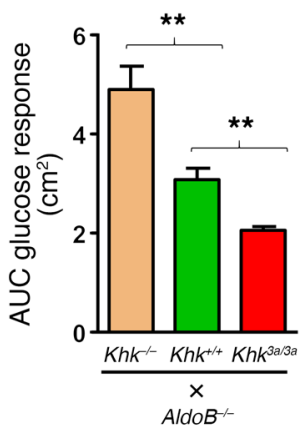

C

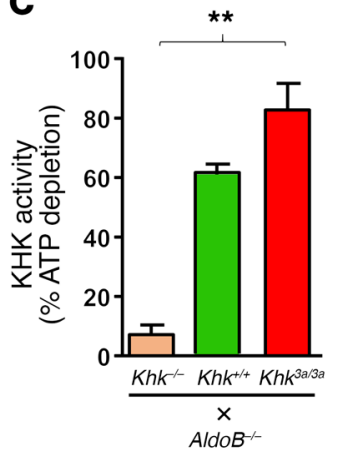

D

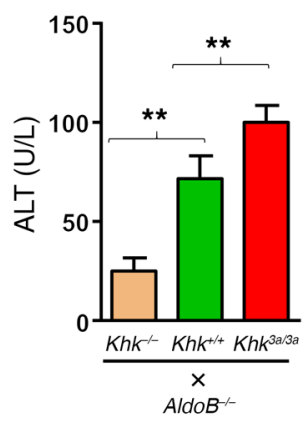

E

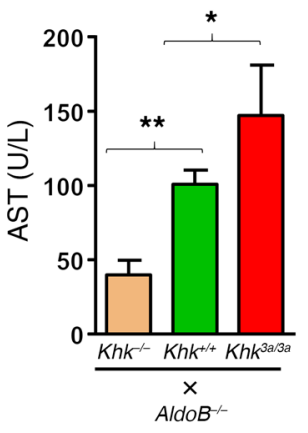

I

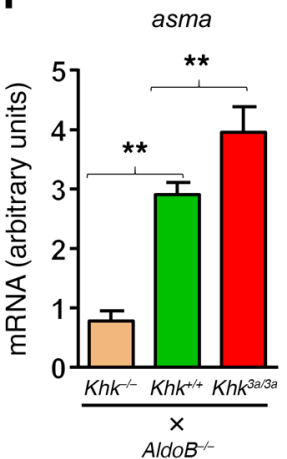

$\mathbf{F}$

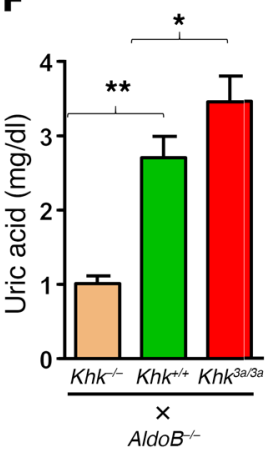

G

H
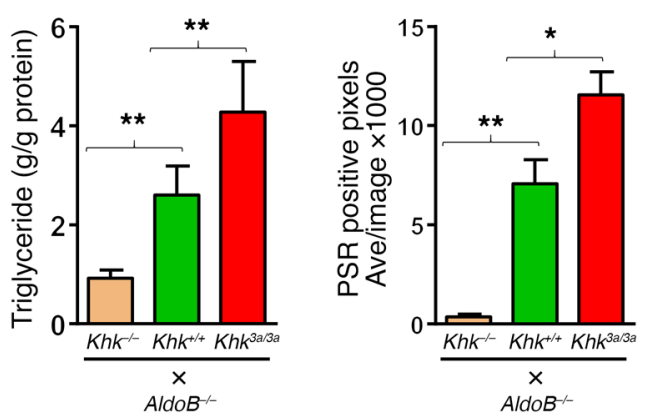

J
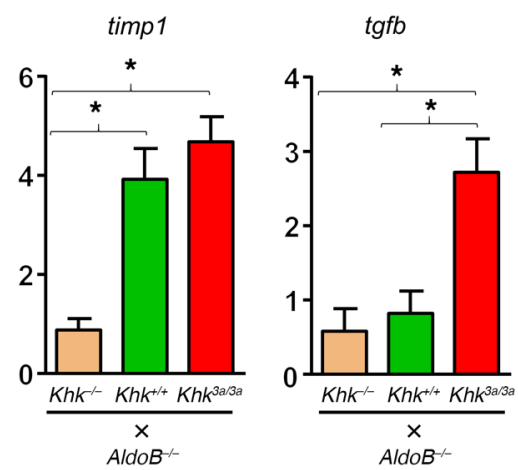

il6

tnfa
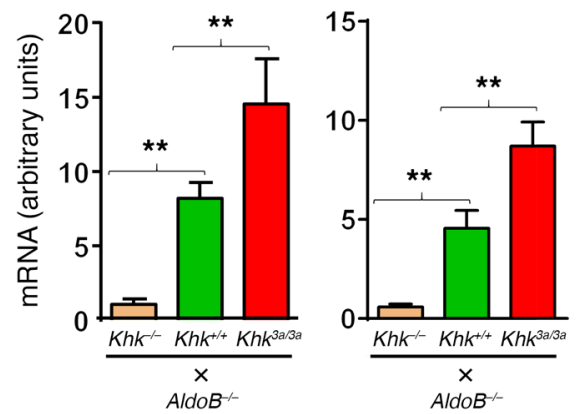

Figure 5. Isoform-specific effects of Khk knockout in the protection of AldoB-KO mice exposed to fructose. (A) Time course of serum glucose levels in

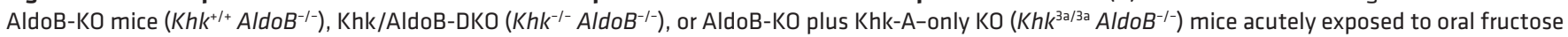
$(1.75 \mathrm{~g} / \mathrm{kg})$. (B) Area under the curve was calculated for the first 40 minutes of serum glucose levels after acute exposure to fructose (1.75 $\mathrm{g} / \mathrm{kg}$ ) for the same animal groups as in A. (C) Khk activity in liver extracts attained 2 hours after fructose exposure for the same animal groups as in A. ATP depletion was calculated versus the baseline for each sample at zero time. (D-F) AST, ALT, and uric acid levels in the serum of the same animal groups as in A. (G) Intrahepatic triglycerides in mice that were chronically fed fructose in the chow (0.3\%). (H and I) Liver fibrosis determined as positive pixel area of PSR staining and mRNA levels of profibrotic genes asma, timp1, and tgfb. (J) mRNA levels of proinflammatory genes //6 and Tnfa. Pairwise statistical significance was calculated using 1-way ANOVA, Tukey's post hoc $t$ test; ${ }^{*} P<0.05$ and ${ }^{* *} P<0.01$ ( $n=75$ animals per group).

shifting of glucose toward glycogen accumulation. Other parameters besides hypoglycemia commonly observed in HFI include hypermagnesemia, hyperuricemia, hypophosphatemia, and transaminitis (25). Serum and liver parameters were analyzed after a 90-minute, $1.75 \mathrm{~g} / \mathrm{kg}$ dose challenge of fructose for WT, AldoB-KO, Khk-KO, and Khk/AldoB-DKO mice. Overall, there was protection from acute changes in the Khk/AldoB-DKO mice, except for elevated fructosemia, which was observed in all mice after fructose exposure (Table 1). Lastly, an inflammatory component was observed in the duodenum and jejunum of fructoseexposed AldoB-KO but not Khk/AldoB-DKO mice. This response was characterized by the presence of apoptotic cells in the intes- tinal lumen and derangement of apical villi (Figure $4 \mathrm{H}$ ), particularly in the duodenum and jejunum corresponding to the intestinal segments that highly express both Khk and aldolase B $(36,37)$.

Khk-A depletion exacerbates the pathophysiology of HFI in AldoB$K O$ mice. Two alternative splice isoforms of the Khk gene, Khk-A and Khk-C, coexist in most mammals, including humans $(23,24)$. Of these isoforms, Khk-C has higher affinity for fructose and thus causes greater ATP reduction in response to a fructose challenge compared with Khk-A (22). The specific knockout of Khk-A (Khk $\left.{ }^{3 \mathrm{a} / 3 \mathrm{a}}\right)$ is associated with an exacerbated metabolic phenotype in mice in response to long-term exposure to fructose (26). Consistent with a role in buffering the overall fructose metabolism in other tissues, 
A

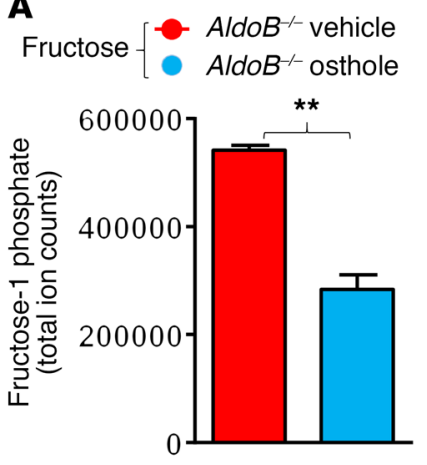

D

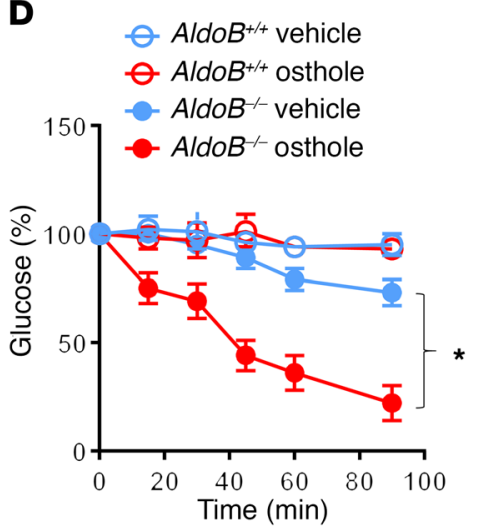

E

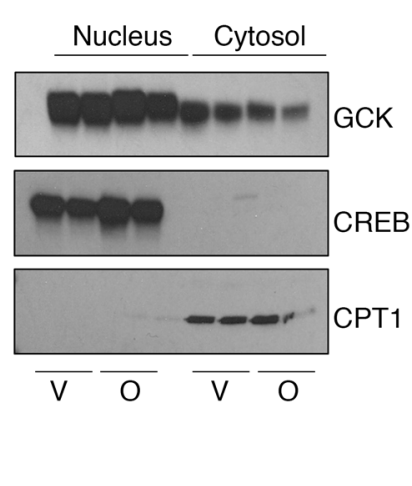

F

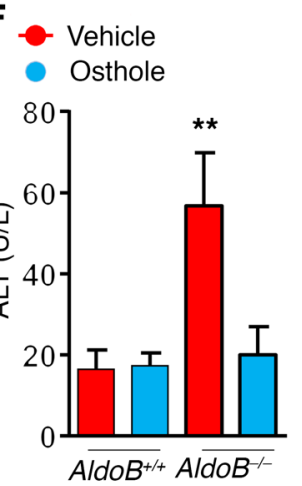

H
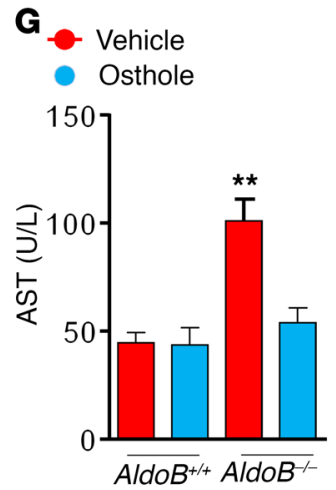

B
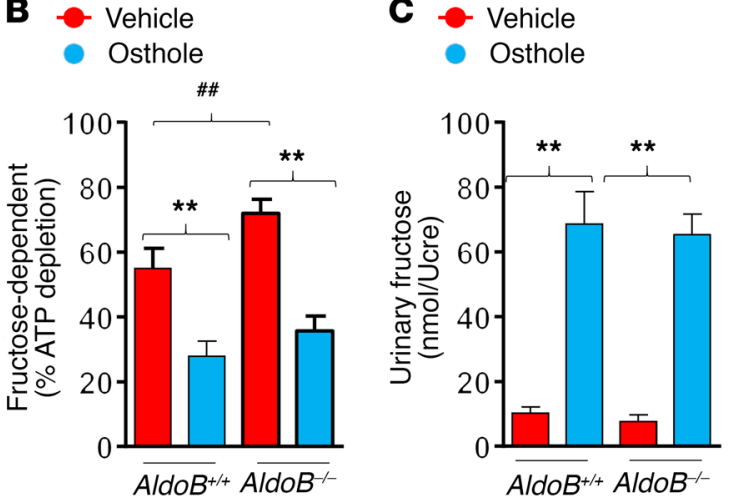

C Vehicle

- Osthole
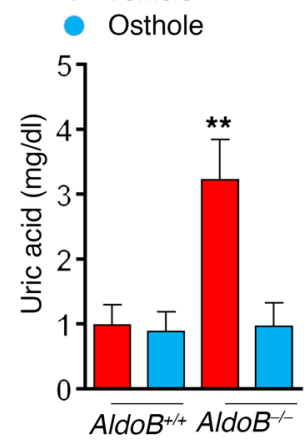

I
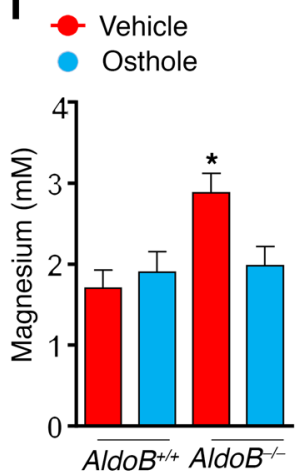

Figure 6. Prophylactic pharmacological inhibition of Khk protects AldoB-KO mice against the deleterious effects of fructose. AldoBKO mice (Aldo $B^{-I}$ ) or WT littermates $\left(\mathrm{AldoB}^{+/+}\right)$were treated with either vehicle (red) or osthole (blue) $(25 \mathrm{mg} /$ $\mathrm{kg}$ ) in the drinking water for 7 days (water was changed twice daily). (A) Fru1-P levels in the liver 90 minutes after fructose challenge. (B) Fructose-dependent ATP depletion attained 2 hours after an acute oral dose of fructose $(1.75 \mathrm{~g} / \mathrm{kg})$. (C) Urinary fructose excretion in the same groups as in $\mathbf{B}$. (D) Time course of serum glucose levels in AldoB-KO mice after acute oral dose of fructose $(1.75 \mathrm{~g} / \mathrm{kg})$. (E) Representative Western blot for glucokinase (GCK) expression in nucleus and cytosol of AldoB-KO mice exposed to fructose for 90 minutes and treated with either vehicle $(V)$ or osthole (O). CREB and CPT1 are markers of the nuclear and cytosolic fractions. (F-I) Serum from AldoB-KO mice attained after acute oral dose of fructose $(1.75 \mathrm{~g} / \mathrm{kg})$ was analyzed for ALT, AST, uric acid, and magnesium. Pairwise statistical significance was calculated using 1-way ANOVA, Tukey's post hoc $t$ test; ${ }^{*} P<0.05$, ${ }^{* *} P<0.01, \# \#<0.05$ ( $\mathrm{n}=7$ animals per group). the Khk-A/AldoB-KO double knockout mice $\left(\mathrm{Khk}^{3 \mathrm{a} / 3 \mathrm{a}} \mathrm{AldoB^{-- }}\right)$ are not protected against the pathology of fructose exposure and had an exacerbated hypoglycemic response in response to fructose compared with AldoB-KO mice (Figure 5, A and B). In parallel, there was a slight increase in liver Khk activity as determined by percentage of ATP depletion (Figure 5C). Perhaps as a result of this increased Khk activity, serum levels of liver AST, ALT, and uric acid were significantly higher in $K h k^{3 a / 3 a} A l d o B^{-/-}$compared with AldoB-KO animals (Figure 5, D-F and Table 1). Furthermore, $K h k^{3 \mathrm{a} / 3 \mathrm{a}} \mathrm{AldoB}^{-/-}$mice that were chronically exposed to low amounts of fructose had significantly greater lipogenic, profibrotic, and proinflammatory hepatic profiles compared with regular AldoB-KO mice (Figure 5, G-J). This study provides evidence supporting the hypothesis that the inhibition of Khk activity in tissues expressing the Khk-C isoform (e.g., the liver) confer protection against HFI in mice.

Effective pharmacological inhibition of Khk for the prevention and treatment of HFI in mice. Osthole, a coumarinic derivative obtained from plants of the Angelica family, has efficient inhibitory activity against Khk (38). To test whether osthole could be used for inhibition of Khk activity in the liver and thus confer protection against HFI in mice, AldoB-KO mice were exposed to osthole $(25 \mathrm{mg} / \mathrm{kg}$, Santa Cruz Biotechnologies, catalog SC-205780) in the drinking water for 7 days before an acute fructose challenge of $(1.75 \mathrm{~g} / \mathrm{kg})$. AldoB-KO mice pretreated with osthole demonstrated significantly reduced Khk activity and Fru1-P accumulation in their livers and less of an ATP decrease compared with vehicle-exposed mice (Figure 6, A and B). Consistently, exposure of mice to osthole resulted in significantly increased urinary fructose excretion compared with vehicle-treated animals, consistent with blocking fructose metabolism (as in essential fructosuria) (Figure 6C). More importantly, monitoring blood glucose for 90 minutes after an acute fructose challenge showed that osthole-treated AldoB-KO mice had significantly improved glycemia (Figure 6D). Consistent with improved glycemia and reduced Fru1-P levels, the release of GCK to the cytosol from the 
nucleus was significantly reduced in osthole-treated mice compared with vehicle-treated mice (Figure 6E). Furthermore, monitoring other serum metabolites 90 minutes after acute fructose challenge showed that osthole-treated mice had reduced transaminitis, hyperuricemia, and hypermagnesemia (Figure 6, F-I). However, it is important to note that osthole is not specifically a fructokinase inhibitor and therefore the beneficial effects of fructokinase may be related to multiple off-target effects (39). Nevertheless, our study suggests that the prophylactic pharmacological inhibition of Khk is therapeutically effective in prevention of acute pathology in this animal model for HFI.

\section{Discussion}

For the first time to our knowledge, evidence is reported herein that the blockade of Khk could be an excellent therapeutic approach for the prevention and treatment of HFI. New therapeutic approaches for HFI are needed because of the increased challenge of excluding fructose and added sugars in the diet due to their ubiquitous use in food preparations $(3,40)$. This issue alone severely affects the quality of life for individuals with HFI, who must be constantly concerned about both acute and chronic exposure to fructose, which leads to severe hypoglycemia and liver disease, respectively. In this regard, by using both the newly created AldoB-KO mice (16) and a newly discovered Khk inhibitor, osthole (24), the multiple deleterious effects that fructose poses for individuals with HFI as well as for this animal model were blocked, including acute hypoglycemic shock, liver inflammation, and intestinal damage.

Moreover, ample recent evidence strongly indicates that the increased consumption of fructose and sugar in recent decades has substantially contributed to the current epidemics of obesity and metabolic syndrome (41-44). It is commonly thought that the association between sugar consumption and metabolic syndrome is merely due to the hypercaloric effect of fructose and sugar as a consequence of excessive intake $(45,46)$. However, there are isocaloric data to the contrary that highlight the peculiarity of fructose and its metabolism $(47,48)$. Importantly, here we show, using AldoB-KO mice, that even when fructose cannot be directly metabolized into triglycerides, glucose, or glycogen, it can induce specific harmful metabolic effects in the organism, including fatty liver and liver inflammation, which would underscore the importance of the particular metabolism of fructose in disease.

Despite complete avoidance of fructose-containing foods, individuals with HFI often develop episodes of hypoglycemia, show signs of general ill health, and exhibit symptoms of chronic fructose intoxication (8). As there is little to no ingestion of fructose, these symptoms must be related to the metabolic and not the caloric properties of fructose. The multi-variant symptoms of chronic fructose intoxication could be from inadvertent ingestion of foods that contain fructose or fructose-containing sugars (sucrose and HFCS), which are heavily added to foods in order to make them tastier and more appealing to consumers. Foods with added sugars are not necessarily sweeter, but this sweetness is a powerful aversion to those with HFI (49). In addition, it has become increasingly apparent that fructose can be produced endogenously in the body by the activation of aldose reductase and the polyol pathway $(50,51)$. These reports demonstrate that endogenous fructose production and its metabolism through Khk are important del- eterious steps in the pathogenesis of fatty liver and steatohepatitis. Consistently, individuals with HFI do not tolerate foods containing sorbitol, an intermediate product of the polyol pathway (52) leading to fructose production. Therefore, given the prevention of pathology by limiting the metabolism of fructose through Khk shown here, further studies employing aldose reductase knockout mice or aldose reductase inhibitors, which are already commercially available (unlike drugs targeting Khk), are warranted to prevent the endogenous production and metabolism of fructose.

The mechanism whereby HFI pathology progresses so rapidly after acute fructose exposure remains unclear. What is clear is that this mechanism likely depends on the phosphate depletion that occurs in its sequestration as Fru1-P. However, substantial ATP depletion and uric acid generation also occur and are exacerbated by the overactivation of Khk (Figure 1). Therefore, substantial ATP depletion and uric acid production and its hepatic accumulation may be yet-unknown underlying factors important in the pathogenesis of HFI. Consistent with this hypothesis, uric acid is an important prooxidant molecule that can cause intracellular oxidative stress, inflammation, and mitochondrial dysfunction (53-56). In this study, inhibiting Khk activity did not reveal the details of the molecular mechanisms linked with the disease, but did reveal the intracellular hallmarks that are part of the pathogenic pathway (e.g., phosphate depletion, ATP depletion, Fru1-P generation, etc.). Given the model organism and the effective cure described here, deciphering this pathway is within reach.

\section{Methods}

Animal study. AldoB-KO and Khk-KO mice in the C57BL/6 background were generated as previously described $(19,24,57)$. Mice were maintained in temperature- and humidity-controlled specific pathogen-free conditions on a 14-hour-dark, 10-hour-light cycle, and allowed ad libitum access to normal laboratory chow (Harlan Teklad, catalog 2920X) (Figure 3 and Figure 5) or a fructose-free diet (Bioserv, catalog F6700) for AldoB-KO mice. AldoB/Khk (lacking both A and $\mathrm{C}$ isoforms of Khk) knockout mice and AldoB/Khk-A knockout mice were developed by crossing F2 heterozygous mice $\left(\mathrm{Khk}^{+/-}\right.$aldo $\mathrm{B}^{+/-}$or $\mathrm{Kh}^{+/ 3 \mathrm{a}}$ aldoB $\left.\mathrm{B}^{+-}\right)$and maintained on a fructose-free diet. All experiments were conducted with adherence to the NIH Guide for the Care and Use of Laboratory Animals. The animal protocol was approved by the Institutional Animal Care and Use Committee of the University of Colorado. In all studies, 8-week-old male mice were used.

An oral fructose tolerance test was performed on 8-week-old, 6-hour-fasting male mice by gavage of a fixed amount of fructose as indicated for each experiment (Figure 4A, Figure 5A, and Figure 6A). Baseline, 15-, 30-, 45-, 60-, and 90-minute glucose levels were determined with a glucometer from tail snips. The AUC for glucose was calculated by arbitrarily providing $1 \mathrm{~cm}$ per $50 \%$ of the glucose level and per 30 minutes of time. Therefore, the disappearance of $50 \%$ of baseline blood glucose in 30 minutes would result in $0.5 \mathrm{~cm}^{2}$ loss in the AUC. Similarly, an oral pyruvate tolerance test was performed in 6-hour-fasting mice exposed to $1.5 \mathrm{~g} / \mathrm{kg}$ pyruvate, and serum glucose was determined over a 120-minute period.

Biochemical analysis. Blood was collected in microtainer tubes (BD Biosciences) from cardiac puncture of mice under isoflurane, and serum was obtained after centrifugation at 13,000 rpm for $2 \mathrm{~min}$ utes at room temperature. Biochemical analysis for ALT, AST, glu- 
cose, and uric acid was performed on serum and liver lysates with an automated chemistry analyzer (VetACE Clinical Chemistry System, Alfa Wassermann Diagnostic Technologies). Serum or liver fructose, phosphate, and magnesium determination was performed biochemically following the manufacturers' instructions (fructose: Bioassay Systems EFRU250; phosphate and magnesium: Biovision K410 and K385). Urinary fructose levels were normalized to units of creatinine, determined biochemically on liver extracts with a Creatinine Reagent Kit (Pointe Scientific). Liver hydroxyproline was determined biochemically following the manufacturer's protocol (Biovision, K555). To extract metabolites from liver samples, frozen liver samples were ground at liquid nitrogen temperature with a Cryomill (Retsch). The resulting tissue powder was weighed $(\sim 20 \mathrm{mg})$. The extraction was then done by adding $-20^{\circ} \mathrm{C}$ extraction solvent to the powder and incubating it at $-20^{\circ} \mathrm{C}$ overnight, followed by vortexing and centrifugation at $16,000 \mathrm{~g}$ for 10 minutes at $4^{\circ} \mathrm{C}$. The volume of the extraction solution $(\mu \mathrm{l})$ was 40 times the weight of tissue $(\mathrm{mg})$ to make an extract of $25 \mathrm{mg}$ tissue per milliliter of solvent. Dried extracts were then redissolved in LC-MS Grade water (catalog 51140; Thermo Fisher). Metabolites were analyzed via reverse-phase ion-pairing chromatography coupled to an Exactive Orbitrap mass spectrometer (Thermo Fisher Scientific). The mass spectrometer was operated in negative-ion mode with a resolving power of 100,000 at a mass-to-charge ratio $(\mathrm{m} / \mathrm{z})$ of 200 and a scan range of 75-1,000 m/z. The liquid chromatography (LC) method used an Atlantis T3 column $(150 \mathrm{~mm} \times 2.1 \mathrm{~mm}$, $3 \mu \mathrm{m}$ particle size, $100 \AA$ A pore size; Waters, catalog 186003719), with a gradient of solvent A (97:3 water/methanol with $10 \mathrm{mM}$ tributylamine and $15 \mathrm{mM}$ acetic acid) and solvent $\mathrm{B}$ (methanol). The LC gradient was as follows: 0 minutes, $0 \% \mathrm{~B}, 200 \mu \mathrm{l} / \mathrm{min} ; 2$ minutes, $0 \% \mathrm{~B}$, $200 \mu \mathrm{l} / \mathrm{min}$; 4 minutes, $20 \%$ B, $200 \mu \mathrm{l} / \mathrm{min}$; 13 minutes, $80 \%$ B, 200 $\mu \mathrm{l} / \mathrm{min} ; 17$ minutes, $100 \% \mathrm{~B}, 200 \mu \mathrm{l} / \mathrm{min} ; 17.5$ minutes, $100 \% \mathrm{~B}, 300$ $\mu \mathrm{l} / \mathrm{min} ; 20$ minutes, $100 \% \mathrm{~B}, 300 \mu \mathrm{l} / \mathrm{min} ; 20.5$ minutes, $0 \% \mathrm{~B}, 300$ $\mu \mathrm{l} / \mathrm{min}$; 24 minutes, $0 \% \mathrm{~B}, 300 \mu \mathrm{l} / \mathrm{min} ; 25$ minutes, $0 \% \mathrm{~B}, 200 \mu \mathrm{l} /$ min. Other LC parameters were as follows: column temperature $25^{\circ} \mathrm{C}$, autosampler temperature $5^{\circ} \mathrm{C}$, injection volume $10 \mu \mathrm{L}$.

Histopathology. Formalin-fixed paraffin-embedded liver and jejunal sections were stained with H\&E or periodic acid-Schiff (PAS). Histological examination was performed as previously described by Orlicky et al (58). For liver injury scoring, the entire cross section of liver including all zones was analyzed from each mouse. Images were captured on an Olympus BX51 microscope equipped with a 4 megapixel Macrofire digital camera (Optronics) using the PictureFrame Application 2.3 (Optronics). Composite images were assembled with the use of Adobe Photoshop. All images in each composite were handled identically. To quantitate the fibrosis, 10 polarized images were made in a tiling fashion across each PSR-stained slide, then quantitated and averaged using the 3I Slidebook program to arrive at the PSR-stained pixels per 100× field for that slide/animal. The criteria used in liver injury scoring are modified from the criteria used by Brunt (59), and as previously modified for mouse liver (47). The criteria used included: (a) liver cell injury (ballooning, acidophil bodies, necrotic cells, pigmented macrophages, megamitochondria, etc); (b) inflammation (lobular inflammation, foci of inflammatory cells, lipogranulomas, portal inflammation, Langhans giant cells, etc.); (c) steatosis (macro and microvesicular steatosis); (d) fibrosis (perisinusoidal, periportal, bridging fibrosis, cirrhosis, etc.); and (e) other features (glycogenated nuclei, mitotic figures, etc.). To score all animals and as described above, we captured 10 images per animal in a tiling fashion across a section of tissue to obtain an average, then we averaged those numbers in the various groups to determine a mean \pm SD. For intestinal analysis, $3-5$ sections of each mouse were analyzed and scored including duodenal, jejunal, ileal, and cecal sections. Score points included loss of papillae tip, luminal presence of dead and apoptotic cells, and leukocyte infiltration.

Western blotting. Protein lysates were prepared from mouse tissue employing MAPK lysis buffer as previously described (60). Protein content was determined by the bicinchoninic acid (BCA) protein assay (Pierce). Total protein $(50 \mu \mathrm{g})$ was separated by SDS-PAGE $(10 \% \mathrm{w} / \mathrm{v})$, and transferred to PVDF membranes (BioRad). Membranes were first blocked for 1 hour at $25^{\circ} \mathrm{C}$ in $4 \%(\mathrm{w} / \mathrm{v}$ ) instant milk dissolved in 0.1 $\%$ Tween-20 Tris-buffered saline (TTBS), and incubated with the following primary rabbit or mouse-raised antibodies (1:1,000 dilution in TTBS): aldolase B (H00000229-A01), GCK (H000026465-B02P), and PEPCK (H00005105-B01P) (Novus Biotechnologies); G6Pase (ab96142, Abcam) and Khk (HPA007040, Sigma); actin (8432), FAS, ACC (3676), p-ACC (Ser79, 11818), AMPK (2603), pAMPK (Thr172, 2535), ACL (4332), CPT1 (12252), GS (3886), and pGS (Ser641 3981) (all from Cell Signaling); and PYGL (15851-1-AP) and ECH1 (113851-AP) (both from Proteintech). Membranes were visualized using an anti-rabbit (7074) or anti-mouse IgG (7076) horseradish peroxidaseconjugated secondary antibody (1:2,000, Cell Signaling) using the HRP Immunstar detection kit (Bio-Rad). Chemiluminescence was recorded with an Image Station $440 \mathrm{CF}$ and the results were analyzed with 1D Image Software (Kodak Digital Science). For real-time PCR, RNA was extracted with the RNeasy kit (Qiagen) and converted to cDNA with the iScript kit (BioRad). Renal levels of il6 and $c c l 2$ were analyzed with specific primers (60).

Ketohexokinase activity. Khk activity was determined as previously described with slight modifications (50). Briefly, liver samples were first homogenized in $20 \mathrm{mM}$ Tris- $\mathrm{HCl}, \mathrm{pH} 7.5,150 \mathrm{mM} \mathrm{KCl}, 1 \mathrm{mM}$ EDTA, and $1 \mathrm{mM}$ DTT using a polytron homogenizer, and centrifuged for 10 minutes at 13,000 rpm at $4^{\circ} \mathrm{C}$. The protein content of the supernatant fraction was quantified with the protein BCA assay, and Khk activity was measured on $50 \mu$ g lysate protein after addition of a buffer to $5 \mathrm{mM}$ fructose in 50 $\mathrm{mM}$ imidazole, $1 \mathrm{M}$ potassium acetate, $\mathrm{pH}$ 5.2, and $1 \mathrm{mM}$ ATP. ATP was measured both before and after a 2-hour incubation at $37^{\circ} \mathrm{C}$ using the ATP determination kit (K354-100, Biovision) as per the manufacturer's instructions. Khk activity was calculated as the ratio between ATP levels at 2 hours versus baseline for each sample at zero time.

Statistics. All numerical data are mean \pm SEM. Independent replicates for each data point $(n)$ are identified in the figure legends. Data graphics and statistical analysis were performed using Prism 5 (GraphPad). Unless otherwise noted, data were analyzed by 1-way ANOVA, Tukey's post hoc $t$ test. A value of $P<0.05$ was regarded as statistically significant. For Figure 3B and assuming not normal distributions of genotypes, data were analyzed by 2 -tailed $\chi^{2}$ test.

Study approval. All animal experiments were conducted with adherence to the Guide for the Care and Use of Laboratory Animals (National Academies Press, 2011). The animal protocol was approved by the University of Colorado's IACUC.

\section{Author contributions}

MAL, MFW, RJJ, and DRT helped designing research studies. MAL and AAH conducted the experiments. MAL, AAH, CC, CJ, 
NL, TM, and MK acquired the data. MAL, CJ, JDR, RJJ, DJO, and DRT analyzed the data.MAL, RJJ, and DRT wrote the manuscript.

\section{Acknowledgments}

This project was supported by NIH grants 1R01DK108859, 1K01DK095930, and 1R03DK105041 (to MAL) and
1R01DK105634 (to RJJ and MAL). CJ is a postdoctoral fellow of the American Diabetes Association.

Address correspondence to: Dean R. Tolan, Department of Biology, Boston University, 5 Cummington Mall, Boston, Massachusetts, USA. Phone: 617.353.5310; Email: tolan@bu.edu.
1. Johnson RJ, et al. Hypothesis: could excessive fructose intake and uric acid cause type 2 diabetes? Endocr Rev. 2009;30(1):96-116.

2. Johnson RJ, Sánchez-Lozada LG, Andrews P, Lanaspa MA. Perspective: a historical and scientific perspective of sugar and its relation with obesity and diabetes. Adv Nutr. 2017;8(3):412-422.

3. Walker RW, Goran MI. Laboratory determined sugar content and composition of commercial infant formulas, baby foods and common grocery items targeted to children. Nutrients. 2015;7(7):5850-5867.

4. Vos MB, et al. Added sugars and cardiovascular disease risk in children: a scientific statement from the American Heart Association. Circulation. 2017;135(19):e1017-e1034.

5 . WHO guidelines approved by the guidelines review committee. Guideline: sugars intake for adults children. Geneva; World Health Organization: 2015.

6. Tolan DR. Molecular basis of hereditary fructose intolerance: mutations and polymorphisms in the human aldolase B gene. Hum Mutat. 1995;6(3):210-218.

7. Coffee EM, Yerkes L, Ewen EP, Zee T, Tolan DR. Increased prevalence of mutant null alleles that cause hereditary fructose intolerance in the American population. J Inherit Metab Dis. 2010;33(1):33-42.

8. Mock DM, Perman JA, Thaler M, Morris RC. Chronic fructose intoxication after infancy in children with hereditary fructose intolerance. A cause of growth retardation. $N$ Engl J Med. 1983;309(13):764-770.

9. Kogut MD, Roe TF, Ng W, Nonnel GN. Fructoseinduced hyperuricemia: observations in normal children and in patients with hereditary fructose intolerance and galactosemia. Pediatr Res. 1975;9(10):774-778

10. Cain AR, Ryman BE. High liver glycogen in hereditary fructose intolerance. Gut. 1971;12(11):929-932.

11. Thurston JH, Jones EM, Hauhart RE. Decrease and inhibition of liver glycogen phosphorylase after fructose. An experimental model for the study of hereditary fructose intolerance. Diabetes. 1974;23(7):597-604.

12. Grégori C, Schapira F, Kahn A, Delpech M, Dreyfus JC. Molecular studies of liver aldolase B in hereditary fructose intolerance using blotting and immunological techniques. Ann Hum Genet. 1982;46(Pt 4):281-292.

13. Nikkila EA, Perheentupa J. Non-esterified fatty acids and fatty liver in hereditary fructose intolerance. Lancet. 1962;2(7268):1280.

14. Odièvre M, Gentil C, Gautier M, Alagille D. Hereditary fructose intolerance in childhood. Diagnosis, management, and course in 55 patients. Am J Dis Child. 1978;132(6):605-608.
15. Bouteldja N, Timson DJ. The biochemical basis of hereditary fructose intolerance. J Inherit Metab Dis. 2010;33(2):105-112.

16. Bonthron DT, Brady N, Donaldson IA, Steinmann B. Molecular basis of essential fructosuria: molecular cloning and mutational analysis of human ketohexokinase (fructokinase). $\mathrm{Hum} \mathrm{Mol}$ Genet. 1994;3(9):1627-1631.

17. Steinitz H, Mizrahy O. Essential fructosuria and hereditary fructose intolerance. $\mathrm{NEnglJ} \mathrm{Med}$. 1969;280(4):222.

18. Laron Z. Essential benign fructosuria. Arch Dis Child. 1961;36:273-277.

19. Oppelt SA, Sennott EM, Tolan DR. Aldolase-B knockout in mice phenocopies hereditary fructose intolerance in humans. Mol Genet Metab. 2015;114(3):445-450.

20. Abdelmalek MF, et al. Higher dietary fructose is associated with impaired hepatic adenosine triphosphate homeostasis in obese individuals with type 2 diabetes. Hepatology. 2012;56(3):952-960.

21. Sánchez-Lozada LG, et al. Comparison of free fructose and glucose to sucrose in the ability to cause fatty liver. Eur J Nutr. 2010;49(1):1-9.

22. Ishimoto $\mathrm{T}$, et al. Opposing effects of fructokinase $\mathrm{C}$ and $\mathrm{A}$ isoforms on fructose-induced metabolic syndrome in mice. Proc Natl Acad Sci US A. 2012;109(11):4320-4325.

23. Diggle $\mathrm{CP}$, et al. Both isoforms of ketohexokinase are dispensable for normal growth and development. Physiol Genomics. 2010;42A(4):235-243.

24. Diggle CP, et al. Ketohexokinase: expression and localization of the principal fructosemetabolizing enzyme. J Histochem Cytochem. 2009;57(8):763-774.

25. Steinmann B, Gitzelmann R. The diagnosis of hereditary fructose intolerance. Helv Paediatr Acta. 1981;36(4):297-316.

26. Tsutsumi K, Tsutsumi R, Daimon M, Numazaki $\mathrm{M}$, Ishikawa K. Tissue-specific and developmentally specific controls involved in rat aldolase B gene expression. Isozymes Curr Top Biol Med Res. 1987;14:177-193.

27. Gouw AS, Clouston AD, Theise ND. Ductular reactions in human liver: diversity at the interface. Hepatology. 2011;54(5):1853-1863.

28. Gentil C, Colin J, Valette AM, Alagille D, Lelong M. Study of carbohydrate metabolism in the course of hereditary fructose intolerance. Attempt at interpretation of hypoglycemia. Rev Fr Etud Clin Biol. 1964;9:596-607.

29. Bollen M, et al. Effect of fructose 1-phosphate on the activation of liver glycogen synthase. Biochem J. 1986;240(1):309-310.

30. Douillard C, Mention K, Dobbelaere D, Wemeau JL, Saudubray JM, Vantyghem MC. Hypoglycaemia related to inherited metabolic diseases in adults. Orphanet J Rare Dis. 2012;7:26.

31. Cicerchi C, et al. Uric acid-dependent inhibition of AMP kinase induces hepatic glucose production in diabetes and starvation: evolutionary implications of the uricase loss in hominids. FASEB J. 2014;28(8):3339-3350.

32. Baerlocher K, Gitzelmann R, Nüssli R, Dumermuth $\mathrm{G}$. Infantile lactic acidosis due to hereditary fructose 1,6-diphosphatase deficiency. Helv Paediatr Acta. 1971;26(5):489-506.

33. Malaisse WJ, Sener A, Mahy M. The stimulussecretion coupling of glucose-induced insulin release. Sorbitol metabolism in isolated islets. Eur JBiochem. 1974;47(2):365-370.

34. Shiota C, Coffey J, Grimsby J, Grippo JF, Magnuson MA. Nuclear import of hepatic glucokinase depends upon glucokinase regulatory protein, whereas export is due to a nuclear export signal sequence in glucokinase. J Biol Chem. 1999;274(52):37125-37130.

35. Vandercammen A, Van Schaftingen E. Species and tissue distribution of the regulatory protein of glucokinase. Biochem J. 1993;294 ( Pt 2):551-556.

36. Espinoza J, Clark SB, Hritz A, Rosensweig NS. Regulation of rat proximal intestinal glycolytic enzyme activity by ileal perfusion with glucose. Gastroenterology. 1976;71(2):295-298.

37. Johnson RJ, et al. Fructokinase, fructans, intestinal permeability, and metabolic syndrome: an equine connection? JEquine Vet Sci. 2013;33(2):120-126.

38. Le MT, et al. Bioactivity-guided identification of botanical inhibitors of ketohexokinase. PLoS ONE. 2016;11(6):e0157458.

39. Zhang ZR, Leung WN, Cheung HY, Chan CW. Osthole: a review on its bioactivities, pharmacological properties, and potential as alternative medicine. Evid Based Complement Alternat Med. 2015;2015:919616.

40. Newens KJ, Walton J. A review of sugar consumption from nationally representative dietary surveys across the world. J Hum Nutr Diet. 2016;29(2):225-240.

41. Malik VS, Hu FB. Fructose and cardiometabolic health: what the evidence from sugar-sweetened beverages tells us. J Am Coll Cardiol. 2015;66(14):1615-1624.

42. Goran MI. Sugar-sweetened beverages, genetic risk, and obesity. $N$ Engl J Med. 2013;368(3):285-286.

43. Chaloupka FJ, Powell LM, Chriqui JF. Sugarsweetened beverages and obesity: the potential impact of public policies. J Policy Anal Manage. 2011;30(3):645-655.

44. Bray GA, Nielsen SJ, Popkin BM. Consumption of high-fructose corn syrup in beverages may play a role in the epidemic of obesity. Am JClin Nutr. 2004;79(4):537-543.

45. Sievenpiper JL, Toronto 3D (Diet, Digestive Tract, Disease) Knowledge Synthesis Clinical Trials Unit. Fructose: where does the truth lie? 
JAm Coll Nutr. 2012;31(3):149-151.

46. Sievenpiper JL, et al. Heterogeneous effects of fructose on blood lipids in individuals with type 2 diabetes: systematic review meta-analysis of experimental trials in humans. Diabetes Care. 2009;32(10):1930-1937.

47. Roncal-Jimenez CA, et al. Sucrose induces fatty liver and pancreatic inflammation in male breeder rats independent of excess energy intake. Metab Clin Exp. 2011;60(9):1259-1270.

48. Lomba A, Milagro FI, García-Díaz DF, Campión J, Marzo F, Martínez JA. A high-sucrose isocaloric pair-fed model induces obesity and impairs NDUFB6 gene function in rat adipose tissue. J Nutrigenet Nutrigenomics. 2009;2(6):267-272.

49. Wegner $\mathrm{H}$. Oral findings in a sugar--free diet: a study on children with hereditary fructose intolerance. Zahn Mund Kieferheilkd Zentralbl. 1980;68(7):706-712.

50. Lanaspa MA, et al. Endogenous fructose production and fructokinase activation mediate renal injury in diabetic nephropathy. J Am Soc Nephrol. 2014;25(11):2526-2538.

51. Lanaspa MA, et al. Endogenous fructose production and metabolism in the liver contributes to the development of metabolic syndrome. Nat Commun. 2013;4:2434.

52. Sachs M, Asskali F, Förster H, Encke A. Repeated perioperative administration of fructose and sorbitol in a female patient with hereditary fructose intolerance (HFI). Z Ernahrungswiss. 1993;32(1):56-66.

53. Kanbay M, et al. Uric acid in metabolic syndrome: from an innocent bystander to a central player. Eur JIntern Med. 2016;29:3-8.

54. Chen W, et al. Uric acid suppresses 1 alpha hydroxylase in vitro in vivo. Metab Clin Exp. 2014;63(1):150-160.

55. Sánchez-Lozada LG, et al. Uric acid-induced endothelial dysfunction is associated with mitochondrial alterations and decreased intracellular ATP concentrations. Nephron Exp Nephrol. 2012;121(3-4):e71-e78.
56. Lanaspa MA, et al. Uric acid induces hepatic steatosis by generation of mitochondrial oxidative stress: potential role in fructose-dependent and -independent fatty liver. J Biol Chem. 2012;287(48):40732-40744.

57. Trinh CH, Asipu A, Bonthron DT, Phillips SE. Structures of alternatively spliced isoforms of human ketohexokinase. Acta Crystallogr D Biol Crystallogr. 2009;65(Pt 3):201-211.

58. Orlicky DJ, et al. Chronic ethanol consumption in mice alters hepatocyte lipid droplet properties. Alcohol Clin Exp Res. 2011;35(6):1020-1033.

59. Lanaspa MA, Almeida NE, Andres-Hernando A, Rivard CJ, Capasso JM, Berl T. The tight junction protein, MUPP1, is up-regulated by hypertonicity is important in the osmotic stress response in kidney cells. Proc Natl Acad Sci U S A. 2007;104(34):13672-13677.

60. Andrés-Hernando A, et al. Splenectomy exacerbates lung injury after ischemic acute kidney injury in mice. Am J Physiol Renal Physiol. 2011;301(4):F907-F916. 